\title{
Enhanced Modal Solutions for Structural Dynamics in Aerothermoelastic Analysis
}

\author{
Nathan J. Falkiewicz* and Carlos E. S. Cesnik \\ University of Michigan, Ann Arbor, Michigan 48109
}

DOI: $10.2514 / 1 . C 034122$

\begin{abstract}
Hypersonic vehicle design and simulation require models that are of low order. Modeling of hypersonic vehicles is complicated due to complex interactions between aerodynamic heating, heat transfer, structural dynamics, and aerodynamics in the hypersonic regime. This work focuses on the development of efficient modal solutions for structural dynamics of hypersonic vehicle structures under transient thermal loads. The problem is outlined, and aerothermoelastic coupling mechanisms are described. A previously developed reduced-order time-domain aerothermoelastic simulation framework is used as the starting point for this study. This paper focuses on two main modeling areas: 1) a surrogate modeling technique is employed for directly updating the generalized stiffness matrix and thermal loads based on the transient temperature distribution, and 2) basis augmentation techniques are employed in order to obtain more accurate solutions for the structural dynamic response. The techniques to be studied are described and applied to a representative hypersonic vehicle all-movable lifting surface.
\end{abstract}

$A$
$a^{(j)}$
$b$
$C$
$C(b, X)$
$c$
$c_{p}$
$\tilde{c}$
$d$
${ }$
$F_{S}$
$F_{T}$
Full
$f_{S}$
$f_{T}$
$G\left(X^{(i)}, X^{(j)}\right)$
$H_{i}$
$h$
$h_{i}$
$K_{G}$
$K_{S}$

\begin{tabular}{|c|c|c|}
\hline$K_{T}$ & $=$ & $\begin{array}{l}\text { thermal conductivity matrix of full system in } \\
\text { physical space }\end{array}$ \\
\hline$K_{S}^{*}$ & $=$ & modified structural stiffness matrix \\
\hline$k_{T}$ & $=$ & $\begin{array}{l}\text { generalized thermal conductivity matrix of } \\
\text { reduced system in modal space }\end{array}$ \\
\hline$k_{S}^{*}$ & $=$ & $\begin{array}{l}\text { generalized stiffness matrix of reduced system in } \\
\text { modal space }\end{array}$ \\
\hline$L$ & $=$ & lower triangular factor in decomposition of $K_{S}^{*}$ \\
\hline$L_{\infty}$ & $=$ & $L_{\infty}$ error \\
\hline$l$ & $=$ & number of augmented mode shapes \\
\hline $1 b_{i}$ & $=$ & $\begin{array}{l}\text { lower bound for } i \text { th proper orthogonal } \\
\text { decomposition modal coordinate }\end{array}$ \\
\hline$M$ & $=$ & Mach number \\
\hline$M_{S}$ & $=$ & $\begin{array}{l}\text { structural mass matrix of full system in physical } \\
\text { space }\end{array}$ \\
\hline$M_{T}$ & $=$ & $\begin{array}{l}\text { thermal capacitance matrix of full system in } \\
\text { physical space }\end{array}$ \\
\hline$m_{S}$ & $=$ & $\begin{array}{l}\text { generalized mass matrix of reduced system in } \\
\text { modal space }\end{array}$ \\
\hline$m_{T}$ & $=$ & $\begin{array}{l}\text { generalized thermal capacitance matrix of } \\
\text { reduced system in modal space }\end{array}$ \\
\hline $\max _{i}$ & $=$ & $\begin{array}{l}\text { maximum value of } i \text { th proper orthogonal } \\
\text { decomposition modal coordinate }\end{array}$ \\
\hline $\min _{i}$ & $=$ & $\begin{array}{l}\text { minimum value of } i \text { th proper orthogonal } \\
\text { decomposition modal coordinate }\end{array}$ \\
\hline$n$ & $=$ & $\begin{array}{l}\text { number of aerothermoelastic simulations used } \\
\text { to generate bounds }\end{array}$ \\
\hline$n_{b}$ & $=$ & $\begin{array}{l}\text { number of basis terms used in kriging regression } \\
\text { model }\end{array}$ \\
\hline$n_{e}$ & $=$ & number of kriging evaluation cases \\
\hline$n_{F}$ & $=$ & $\begin{array}{l}\text { number of specified load vectors in load- } \\
\text { dependent Ritz vector algorithm }\end{array}$ \\
\hline$n_{i v}$ & $=$ & number of input variables to kriging model \\
\hline$n_{k}$ & $=$ & number of kriging sample points \\
\hline$n_{\mathrm{POD}}$ & $=$ & $\begin{array}{l}\text { number of proper orthogonal decomposition } \\
\text { snapshots }\end{array}$ \\
\hline$n_{p}$ & $=$ & number of output parameters in kriging snapshot \\
\hline$n_{R}$ & $=$ & $\begin{array}{l}\text { number of load-dependent Ritz vectors per } \\
\text { specified load vector }\end{array}$ \\
\hline$n_{V}$ & $=$ & $\begin{array}{l}\text { number of free vibration modes used in } \\
\text { structural basis }\end{array}$ \\
\hline $\mathcal{O}$ & $=$ & order of kriging regression model \\
\hline$p_{k}$ & $=$ & kriging fitting parameter in correlation function \\
\hline$q$ & $=$ & number of kriging output quantities \\
\hline$R$ & $=$ & kriging regression function \\
\hline ROM & $=$ & solution vector of reduced-order model \\
\hline$R_{x}$ & $=$ & $\begin{array}{l}\text { matrix of kriging basis functions evaluated at } \\
\text { each snapshot }\end{array}$ \\
\hline
\end{tabular}

Presented as Paper 2011-1963 at the 13th AIAA Dynamic Specialist Conference, Denver, CO, 4-7 April 2011; received 11 July 2016; accepted for publication 27 July 2016; published online 10 November 2016. Copyright $(\odot)$ 2016 by Nathan J. Falkiewicz and Carlos E. S. Cesnik. Published by the American Institute of Aeronautics and Astronautics, Inc., with permission. All requests for copying and permission to reprint should be submitted to CCC at www.copyright.com; employ the ISSN 0021-8669 (print) or 1533-3868 (online) to initiate your request. See also AIAA Rights and Permissions www. aiaa.org/randp.

*Ph.D. Candidate, Department of Aerospace Engineering, 1320 Beal Ave.; currently Technical Staff, Lincoln Laboratory, Massachusetts Institute of Technology, Lexington, MA 02420. Senior Member AIAA.

†Professor of Aerospace Engineering, Department of Aerospace Engineering, 1320 Beal Ave. Fellow AIAA.
$=$ snapshot matrix

$=$ correlation matrix

= vector of proper orthogonal decomposition modal coordinates

vector of proper orthogonal decomposition modal coordinates for transformed system point

modulus of elasticity space

thermal load vector of full system in physical

solution vector of full-order model

generalized structural load vector of reduced generalized thermal load vector of reduced system in modal space

- Gaussian correlation function for kriging model structural response

$=$ altitude

$=$ geometric stiffness matrix

$=$ structural stiffness matrix 


\section{Subscripts}

$\begin{array}{ll}\mathrm{AE} & =\text { aeroelastic } \\ \mathrm{AT} & =\text { aerothermal } \\ \mathrm{HT} & =\text { heat transfer } \\ h & =\text { heated } \\ I & =\text { user-determined input } \\ L & =\text { lower bound } \\ \max & =\text { maximum application temperature } \\ U & =\text { upper bound } \\ u & =\text { unheated } \\ 0 & =\text { initial }\end{array}$

\section{Superscripts}

$=$ number of degrees of freedom of reduced system $=$ vector of kriging regression functions

$=$ number of degrees of freedom of full-order system

$=$ vector of discrete nodal temperatures

$=$ scalar value of uniform initial temperature distribution

$=$ excess of temperature over initial conditions

$=$ time

$=$ time to evaluate kriging model

$=$ time to generate kriging model

$=$ time to generate kriging training data

$=$ upper triangular matrix

$=$ upper bound for $i$ th proper orthogonal decomposition modal coordinate

$=$ input variable to function

$=$ matrix of kriging sample points for training data

$=$ structural degrees of freedom in physical space

$=$ snapshot vector/matrix of training data for kriging model

$=i$ th entry of response vector for $j$ th kriging snapshot

$=$ kriging approximation to function at point $b$

$=$ realization of stochastic process with zero mean and variance $\sigma^{2}$

$=$ stochastic process vector evaluated at each kriging snapshots

$=$ angle of attack

$=$ coefficient of thermal expansion

$=$ coefficients of basis functions in kriging regression model

$=$ time-step size

$=$ kriging fitting parameters in correlation function

$=$ thermal conductivity of material

$=$ Poisson's ratio

$=$ density of material

$=$ variance

$=$ modal matrix of structural reference modes

$=$ modal matrix of full set of thermal proper orthogonal decomposition basis vectors

$=$ modal matrix of truncated set of proper orthogonal decomposition basis vectors

$=j$ th free vibration mode

$=j$ th thermal basis vector

$=i$ th load-dependent Ritz vector

$=i$ th load-dependent Ritz vector before normalization

\begin{tabular}{|c|c|c|}
\hline$A$ & $=$ & $\begin{array}{l}\text { component of structural load vector due to } \\
\text { aerodynamic pressure }\end{array}$ \\
\hline$a$ & $=$ & approximate \\
\hline$H$ & $=$ & $\begin{array}{l}\text { component of structural load vector due to } \\
\text { heating }\end{array}$ \\
\hline$(n)$ & $=$ & time level \\
\hline$T$ & $=$ & matrix transpose \\
\hline-1 & $=$ & matrix inverse \\
\hline
\end{tabular}

\section{Introduction}

D ESIGN and simulation of hypersonic vehicles (HSVs) require consideration of a variety of disciplines due to the highly coupled nature of their flight regime [1]. To capture all of the potential effects on vehicle dynamics, one must consider the aerodynamics, aerodynamic heating, heat transfer, and elastic airframe, as well as the interactions between these disciplines. The problem is further complicated by the large computational expense involved in capturing all of these effects and their interactions in a full-order sense. Although high-fidelity modeling techniques exist for each of these disciplines, the use of such techniques is computationally infeasible in a vehicle design and simulation setting for such a highly coupled problem. Early in the design stage, many iterations of analyses may need to be carried out as the vehicle design matures, thus requiring quick analysis turnaround times. Additionally, the number of states and number of degrees of freedom used in the analyses must be small enough to allow for efficient control simulation and design. As a result, alternative approaches must be considered for vehicle simulations.

There are two methodologies that can be used in the generation of low-order models. The first approach is to apply assumptions that enable the use of simplified models. These models are characterized by their low-order form, and they can often be solved analytically. Although these models are useful in generating a low-order representation of the physics, the simplifying assumptions made to employ these models often preclude the ability to model detailed geometries or complex physics. This work therefore makes use of an alternative approach that involves the use of reduced-order models (ROMs) that are derived from high-fidelity analysis tools. Use of high-fidelity tools alone is infeasible due to their high order and long runtime. Thus, this study seeks to go beyond simply coupling existing high-fidelity codes and routines, and it instead proposes to use the output of these tools along with model reduction techniques to generate computationally tractable systems of governing equations. The objective of the current work is to make the aerothermoelastic (ATE) simulation of complex geometries feasible without the need for a priori assumptions regarding the physics of the problem. As opposed to a simplified modeling approach, by first modeling as much of the physics as possible and then systematically reducing the order of the system, we can control and quantify the error incurred through model reduction. This also allows for tailoring of the number of states and degrees of freedom, as different levels of fidelity may be required as the vehicle configuration matures.

\section{Previous Work on Aerothermoelastic Modeling}

Recent work on reduced-order structural dynamic modeling has focused largely on modal methods to reduce the size of the problem. In two works associated with the geometrically nonlinear thermoelastodynamic response of panels [2,3], the structural basis was composed as a set of linear modes appended with an ensemble of "dual" or "companion" modes, which are associated with the inplane displacements induced by the transverse modes [4]. In both [2] and [3], the dual modes were computed by performing a series of nonlinear static solutions at specific loading conditions. Both works demonstrated the ability of the methodology to capture the geometrically nonlinear response of panels under thermal and acoustic loading.

Another philosophy that has been used to obtain reduced-order solutions to nonlinear vibration problems involves employing system identification techniques to guide the modal basis selection [5-8]. In [8], system identification of a nonlinear system was first performed using proper orthogonal decomposition and smooth orthogonal decomposition [9] in order to identify the dominant proper orthogonal modes of the system. The modal assurance criterion or modal expansion theorem was then invoked to determine the linear normal mode corresponding to each dominant proper orthogonal mode. Once the basis was assembled, it was applied to the nonlinear equations of motion (EOMs) in order to reduce the size of the system. Application of the methodology to a rectangular panel demonstrated the ability of the formulation to select an accurate and efficient basis 
for the simulation. As opposed to previous efforts, the approach used in [8] did not require separate analyses for the transverse and in-plane displacement components.

Although the aforementioned works provided progress in the area of reduced-order modeling, efforts to employ reduced-order models within a fully coupled aerothermoelastic framework have been limited. Additionally, in much of the thermoelastic reduced-order modeling literature, either the temperatures or the heat flux loads on the structure were prescribed a priori. In HSV applications, the thermal boundary conditions (BCs) are not known a priori, as they depend on the aerodynamic flow properties and the wall temperature of the structure. Thus, the structural dynamic and thermal ROMs must be coupled such that they capture this interdependence.

The thermoelastic portion of the current work is a continuation of previous studies on reduced-order modeling of the heat transfer and structural dynamics problems [10-13]. The thermoelastic ROM developed by the authors was combined with an aerothermal ROM [14] into a unified unsteady aerothermoelastic framework [13]. It used reduced-order structural dynamic, heat transfer, and aerodynamic heating models in a time-marching simulation framework. The structural and thermal modes were used to parameterize the structural dynamic response and wall temperature to allow for discretization of the parameter space and enable creation of the aerothermal kriging surrogate. Note that the structural ROM required the greatest amount of computational time of the various components of the solution in [13].

This paper focuses on the use of reduced-order structural dynamic and thermal models within a fully coupled aerothermoelastic framework. Specific challenges associated with using such ROMs in an integrated framework are discussed, and methodologies are developed to address those challenges. One objective of the current study is to improve the computational efficiency of the structural ROM by developing the capability to directly update the generalized stiffness and thermal loads as a function of temperature. Another objective of this paper is to improve the accuracy of the structural ROM via basis augmentation.

\section{Fundamental Aerothermoelastic Problem}

The progress described previously has led to further investigation into the aerothermoelastic coupling exhibited in hypersonic flight. A flowchart of the overall framework applied in this work is shown in Fig. 1. The process begins with the calculation of the heat flux on the outer surface of the structure at the initial time. With the boundary conditions and initial conditions of the thermal problem known, the transient temperature distribution is marched forward in time. A solution of the heat transfer problem is carried out in modal space using modes from proper orthogonal decomposition (POD) to avoid the computational cost of running a full-order finite element analysis. This work considers two coupling mechanisms between the thermal solution and structural stiffness. The first involves geometric stiffening due to thermal stresses that occur in the structure due to differential thermal expansion resulting from the spatially varying temperature distribution. The second is due to the temperature dependence of the Young's modulus resulting from the high temperatures experienced in hypersonic flight. In addition to the thermal effects on the stiffness, the change in temperature also results in thermal loads being applied to the structure. With the stiffness and structural loads known, the structural dynamics system of equations in physical space is transformed to a suitable reduced modal basis, to be described in a subsequent section. The reduced modal system is then solved for the modal coordinates to obtain the structural response. With the deformed configuration known at the current time step, the unsteady aerodynamic flow properties are updated and the process is repeated at the next time step. After a predetermined number of aeroelastic iterations have been carried out, the heat flux boundary conditions are recalculated and the thermal solution is updated.

A time-marching procedure with updates to the thermal and structural boundary conditions at specified intervals is proposed for solution of the coupled aerothermoelastic problem. An outline of the time-stepping schedule is given in Fig. 2. The size of the aeroelastic time step $\Delta t_{\mathrm{AE}}$ is smaller than the size of the aerothermal time step $\Delta t_{\mathrm{AT}}$ due to the fact that the aeroelastic timescale is faster than the thermal timescale. The procedure begins by calculating the aerodynamic flow properties over the undeformed structure at initial time $t_{0}$. Using the flow properties, the heat flux at the outer surface is found along with the local skin-friction coefficients. The aerodynamic pressures and viscous drag components are then integrated to determine the aerodynamic forces and moments at initial time. With the thermal boundary conditions known, a predetermined number of thermal time steps are taken, each of size $\Delta t_{\mathrm{HT}}$, until the time $t_{0}+\Delta t_{\mathrm{AT}}$ is reached. The thermal loads based on the temperature change between $t_{0}$ and $t_{0}+\Delta t_{\mathrm{AE}}$ are then applied to the structural configuration at $t_{0}$. Additionally, the aerodynamic loads based on the already calculated flow properties are applied to the structure. The structural dynamic response solution is then marched forward one time step of size $\Delta t_{\mathrm{AE}}$. The displacements are fed back into the aerodynamic solver, and the flow properties are calculated at time $t_{0}+\Delta t_{\mathrm{AE}}$ over the updated deformed configuration. Each time the flow properties are recalculated, the aerodynamic pressures are also integrated to allow for characterization of the transient aerodynamic forces and moments on the vehicle. The aeroelastic iterations continue to be carried for a predetermined number of time

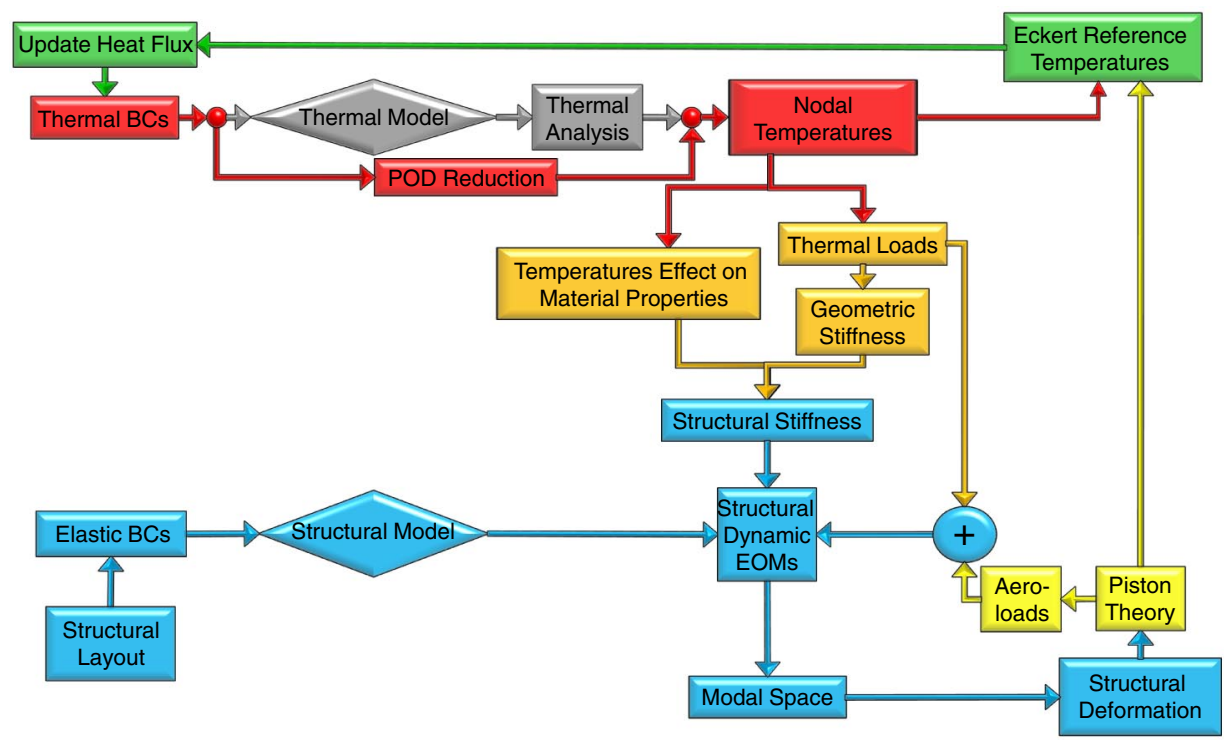

Fig. 1 Reduced-order aerothermoelastic modeling framework. 


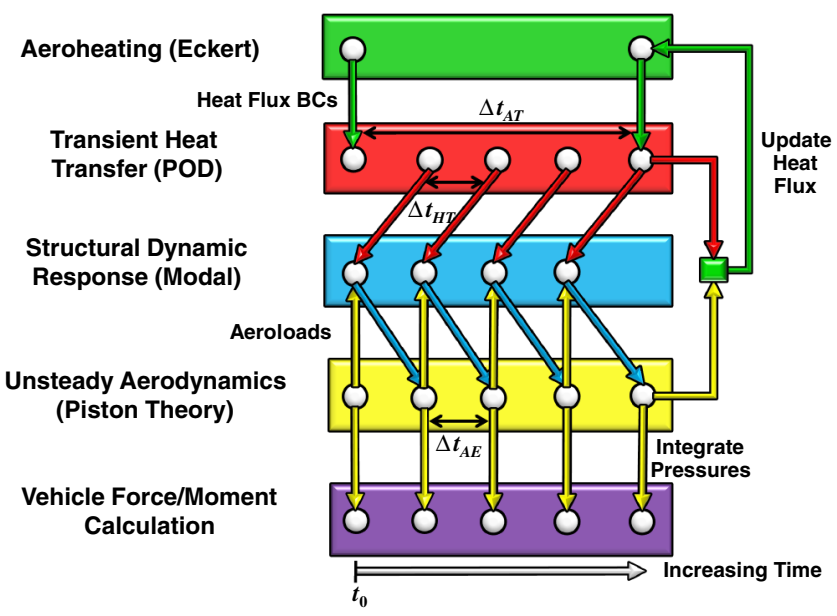

Fig. 2 Overview of aerothermoelastic time-stepping schedule.

steps. Once the time instant $t_{0}+\Delta t_{\mathrm{AT}}$ has been reached, the instantaneous flow properties and wall temperatures are used to update the heat flux boundary conditions to the thermal problem. With the updated thermal boundary conditions known, the transient thermal solution is marched forward from the time instant $t_{0}+\Delta t_{\mathrm{AT}}$ to the time instant $t_{0}+2 \Delta t_{\mathrm{AT}}$, and the process is repeated.

\section{Overview of ROM Generation Process}

As some of the ROMs used in the aerothermoelastic simulation process are dependent on components of other ROMs, these models must be generated in a specific order. An overview of the process used for generating the thermoelastic ROM is given in Fig. 3. The first ROM to be created is the POD model for the transient thermal component of the solution. To begin, the range of vehicle flight parameters is defined. Based on these parameters, representative simulations are carried out and the POD snapshots are extracted. The structural dynamic ROM is dependent on the thermal ROM, and thus the high-fidelity structural model is used in these simulations. The accuracy of the thermal ROM is evaluated by running representative aerothermoelastic simulations using both the thermal ROM and full-

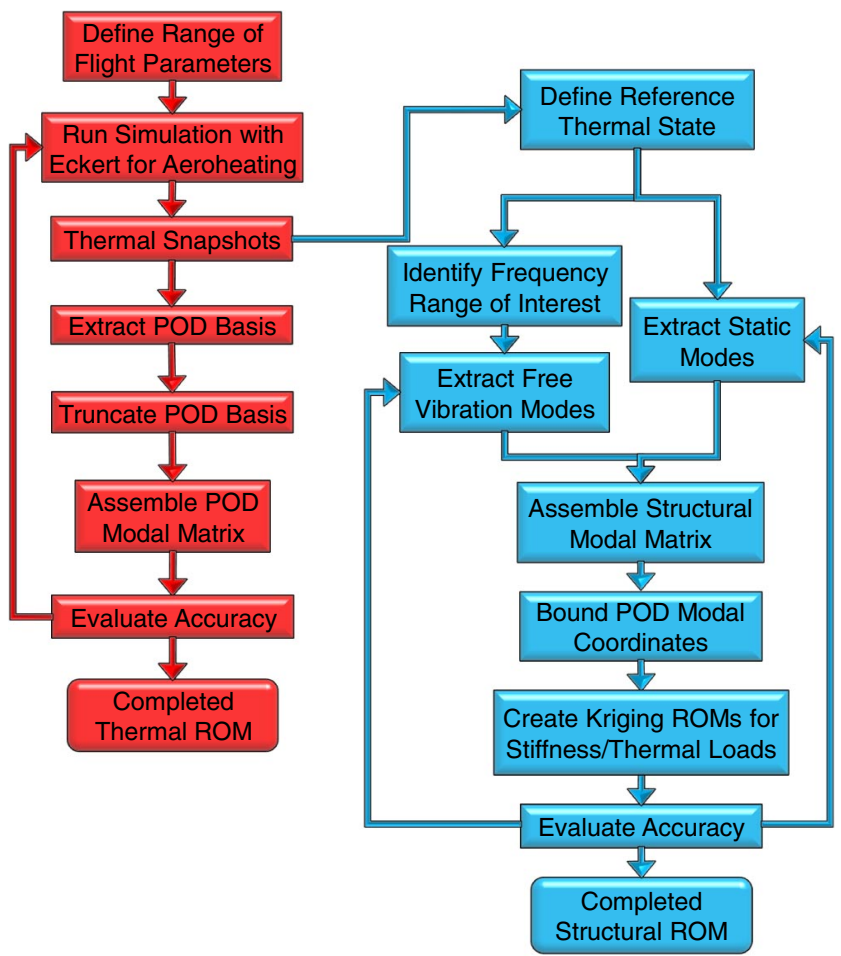

Fig. 3 Flowchart of thermoelastic ROM generation process. order thermal model and comparing their output. If greater accuracy is desired, more snapshots are taken and the thermal ROM is updated.

Once an accurate thermal ROM has been created, the next step is to identify the reference thermal state at which to evaluate the structural free vibration modes. The philosophy used in this work is to take the reference thermal state to be the average nodal temperatures over the thermal snapshots. Based on the frequency range of interest, a set of free vibration modes is evaluated at the reference thermal state, including both temperature-dependent material properties and geometric stiffening due to thermal stresses. Additional modes known as load-dependent Ritz vectors (to be described in a later section) are computed and appended to the structural basis. To avoid the need to reassemble the temperature-dependent stiffness matrix and thermal load vector as the temperature distribution evolves, kriging ROMs of the stiffness matrix and thermal load vector (described in Sec. VI) are created. To generate these ROMs, bounds on the POD modal coordinates are established and kriging training cases are run. At this point, the accuracy of the structural ROM is assessed by again running representative aerothermoelastic simulations and comparing the output of the structural ROM with that of the full-order structural model. If greater accuracy is desired, the structural modal basis is updated and the process is repeated.

\section{Reduced-Order Modeling Formulations}

\section{A. Proper Orthogonal Decomposition for Reduced-Order Thermal} Solution

This work makes use of POD for reduced-order solution of the transient thermal problem. A detailed description of the POD formulation was given in a previous work [12], and thus only a brief overview is presented here. The method of snapshots [15] is used for determination of the POD basis vectors. In this case, the snapshots are defined as vectors of nodal temperatures at various time instants and are computed from a high-fidelity finite element analysis. The goal of the POD formulation is to express the vector of nodal temperatures $T$ at any time instant as a linear combination of the basis, $\varphi(x, y, z)$, with coefficients $c(t)$, i.e.,

$$
\left\{\begin{array}{c}
T_{1}(t) \\
\vdots \\
T_{s}(t)
\end{array}\right\}=\boldsymbol{c}_{1}(t)\left\{\begin{array}{c}
\varphi_{1}^{(1)} \\
\vdots \\
\varphi_{s}^{(1)}
\end{array}\right\}+\boldsymbol{c}_{2}(t)\left\{\begin{array}{c}
\varphi_{1}^{(2)} \\
\vdots \\
\varphi_{s}^{(2)}
\end{array}\right\}+\cdots+\boldsymbol{c}_{\boldsymbol{r}}(t)\left\{\begin{array}{c}
\varphi_{1}^{(r)} \\
\vdots \\
\varphi_{s}^{(r)}
\end{array}\right\}
$$

where $s$ is the total number of degrees of freedom in the finite element model, and $\boldsymbol{r}$ is the total number of POD basis vectors retained after truncation. The basis is computed by first generating the snapshot matrix $A$ given by

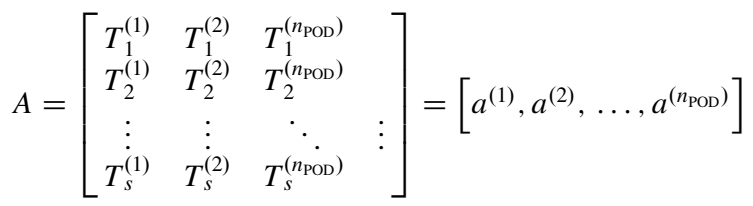

where $T_{i}^{(j)}$ indicates the $i$ th entry of the $j$ th snapshot, $n_{\mathrm{POD}}$ is the number of snapshots taken, and $a^{(j)}$ refers to the column vector corresponding to the $j$ th snapshot. An eigendecomposition or singular value decomposition is used to obtain the POD modal matrix $\Phi_{T}$. Based on the eigenvalues corresponding to each of the POD modes, the dominant POD modes are identified and the truncated POD modal matrix $\bar{\Phi}_{T}$ is formed.

The advantage of using POD is that the computationally intensive process of generating the basis is carried out a priori, and the basis is not updated throughout the simulation. Once the basis has been created, it is applied to the full-order system of heat transfer equations in order to reduce its size. Consider the full-order system of first-order coupled ordinary differential equations governing transient heat transfer:

$$
M_{T} \dot{T}(t)+K_{T} T(t)=F_{T}(t)
$$


where $M_{T}$ is the thermal capacitance matrix, $K_{T}$ is the thermal conductivity matrix, and $F_{T}$ is the thermal load vector. The first step of the reduction process is to project the full-order system onto the truncated POD basis and transform from physical space to modal space using

$$
\bar{\Phi}_{T}^{T} M_{T} \bar{\Phi}_{T} \dot{c}(t)+\bar{\Phi}_{T}^{T} K_{T} \bar{\Phi}_{T} c(t)=\bar{\Phi}_{T}^{T} F_{T}(t)
$$

The generalized thermal capacitance matrix $m_{T}$, generalized thermal conductivity matrix $k_{T}$, and generalized load vector $f_{T}$ are then identified such that

$$
\begin{gathered}
m_{T}=\bar{\Phi}_{T}^{T} M_{T} \bar{\Phi}_{T} \\
k_{T}=\bar{\Phi}_{T}^{T} K_{T} \bar{\Phi}_{T} \\
f_{T}(t)=\bar{\Phi}_{T}^{T} F_{T}(t)
\end{gathered}
$$

Note that, because the POD basis vectors are not eigenvectors of the generalized eigenvalue problem, $m_{T}$ and $k_{T}$ are not diagonal at this stage. Thus, the coupled system is integrated numerically in modal space using a second-order Crank-Nicolson scheme as described in a previous work by the authors [12].

\section{B. Reduced-Order Modal Basis Solution for Structural Dynamic Response}

Although the full-order system of structural dynamic equations of motion is used for comparison purposes, its solution within the aerothermoelastic framework presented here is not suitable for vehicle design and control analysis purposes. The problem of solving for the structural dynamic response of hypersonic vehicle structures within a design and simulation framework is complicated by various factors. Due to the large number of degrees of freedom involved in a traditional finite element solution, steps must be taken to reduce the order of the structural dynamics system of equations. A common approach is to employ a modal transformation in which the structural displacements are expressed as a linear combination of a small number of basis vectors that are the free vibration mode shapes of the structure. However, this approach cannot be applied directly for hypersonic vehicle applications because the mode shapes change over time due to modification of the stiffness from geometric stiffness and temperaturedependent material property effects. The approach taken in this work is to first perform an offline calculation and select a reduced number of Ritz modes onto which the equations of motion are projected. These Ritz modes are then used as the modal basis for solution of the structural response throughout the simulation. This procedure is applicable because the Ritz modes need only to satisfy the geometric boundary conditions [16], which will always be the case, regardless of the stiffness distribution. The modal matrix containing the structural reference modes $\Phi_{S}$ is not updated throughout the simulation, thus preventing the need to recompute the modes during the course of the simulation. Though the reference modes are not updated throughout the simulation, the stiffness matrix is updated each time the structural dynamic response is calculated to account for its dependence on temperature. Updating of the conventional stiffness matrix is performed using the temperature dependence of the material properties of the various materials. The geometric stiffness matrix is updated by solving a static finite element problem based on the thermal loads from temperatures at the current time step and the material coefficients of thermal expansion to calculate the internal loads.

The full-order system of structural dynamic equations of motion in physical space is given by

$$
M_{S} \ddot{x}(t)+K_{S}^{*}(T) x(t)=F_{S}^{H}(t)+F_{S}^{A}(t)
$$

where $M_{S}$ is the mass matrix, $F_{S}^{H}$ is the load vector due to heating, $F_{S}^{A}$ is the load vector due to aerodynamic pressure, and $x$ are the physical degrees of freedom. Note that $F_{S}^{A}$ contains a normal pressure component calculated using a third-order piston theory formulation as well as a wall shear-stress component calculated using the local element skin-friction coefficients obtained from an Eckert reference temperature computation. The modified stiffness matrix $K_{S}^{*}$ is given by

$$
K_{S}^{*}(T) \equiv K_{S}(T)+K_{G}(T)
$$

where $K_{S}(T)$ is the conventional stiffness matrix that varies due to the temperature dependence of the material properties, and $K_{G}(T)$ is the geometric stiffness matrix resulting from thermal stresses. The reduced-order system is obtained by first expressing an approximation to the physical degrees of freedom $x^{a}(t)$ as a linear combination of the structural basis vectors such that

$$
x^{a}(t)=\Phi_{S} d(t)
$$

where $d$ represents the modal coordinates of the reference modes, which are stored as columns of the modal matrix $\Phi_{S}$. Note that, because the number of reference modes used in the modal expansion is much less than the number of physical degrees of freedom in the model, the computational cost of the numerical solution of the system is relatively inexpensive. Once the modified stiffness matrix is known at the current time instant, the system is reduced by substituting Eq. (ㅁ) into Eq. (6) and premultiplying the system by $\Phi_{S}^{T}$, i.e.,

$$
\Phi_{S}^{T} M_{S} \Phi_{S} \ddot{d}(t)+\Phi_{S}^{T} K_{S}^{*}(T) \Phi_{S} d(t)=\Phi_{S}^{T}\left[F_{S}^{H}(t)+F_{S}^{A}(t)\right]
$$

The generalized mass matrix $m_{S}$, generalized stiffness matrix $k_{S}^{*}$, generalized load vector due to heating $f_{S}^{H}$, and generalized load vector due to aerodynamic pressure $f_{S}^{A}$ are then given by

$$
\begin{gathered}
m_{S}=\Phi_{S}^{T} M_{S} \Phi_{S} \\
k_{S}^{*}(T)=\Phi_{S}^{T} K_{S}^{*}(T) \Phi_{S} \\
f_{S}^{H}(t)=\Phi_{S}^{T} F_{S}^{H}(t) \\
f_{S}^{A}(t)=\Phi_{S}^{T} F_{S}^{A}(t)
\end{gathered}
$$

As the mass of the structure is taken to be constant in this work, the reference modes are orthogonal with respect to the mass matrix and the generalized mass matrix $m_{S}$ reduces to the identity matrix.

Since the modified stiffness matrix is continuously changing, we have no guarantee of orthogonality of the reference modes with respect to stiffness, and the equations are coupled. As such, the reduced-order system of equations in modal space is integrated numerically to calculate the vector of modal coordinates at each time instant. As the high-fidelity structural dynamic response solution is treated as the truth model, the numerical integration scheme used for the high-fidelity model (based on Nastran Sol 109) is implemented for solution of the reduced-order system for the modal coordinates $d(t)$ to eliminate any discrepancies in the response due to differences in numerical integration schemes. The numerical integration method is similar to the Newmark- $\beta$ method, except that the load vector is averaged over three time instants and the stiffness matrix is modified such that the dynamic equation of motion reduces to a static solution if no inertial effect or damping exists [17]. The scheme uses a central finite difference representation for the velocity and acceleration at discrete times, given by [17]

$$
\begin{gathered}
\dot{d}^{(n)}=\frac{1}{2 \Delta t_{\mathrm{AE}}}\left(d^{(n+1)}-d^{(n-1)}\right) \\
\ddot{d}^{(n)}=\frac{1}{\Delta t_{\mathrm{AE}}^{2}}\left(d^{(n+1)}-2 d^{(n)}+d^{(n-1)}\right)
\end{gathered}
$$

where the superscript $(n)$ refers to time. The initial conditions, $d^{(0)}$ and $\dot{d}^{(0)}$, are used to generate the vectors $d^{(n-1)}, f_{S}^{(n-1)}$, and $f_{S}^{(n)}$ for the initial time step $(n=0)$ using 


$$
\begin{gathered}
\dot{d}^{(-1)}=d^{(0)}-\dot{d}^{(0)} \Delta t_{\mathrm{AE}} \\
f_{S}^{(-1)}=k_{S}^{*} d^{(-1)} \\
f_{S}^{(0)}=k_{S}^{*} d^{(0)}
\end{gathered}
$$

Note that this formulation assumes that the initial acceleration for all points is zero (initial velocity is constant). Substituting the finite difference approximations of the velocity and accelerations [Eqs. (11)] into the equations of motion [Eq. (6)] and averaging the applied loads over three adjacent time instants, the equations of motion are rewritten as

$$
H_{1} d^{(n+1)}=H_{2}+H_{3} d^{(n)}+H_{4} d^{(n-1)}
$$

where

$$
\begin{gathered}
H_{1}=\frac{1}{\Delta t_{\mathrm{AE}}^{2}} m_{S}+\frac{1}{3} k_{S}^{*} \\
H_{2}=\frac{1}{3}\left(f_{S}^{(n+1)}+f_{S}^{(n)}+f_{S}^{(n-1)}\right) \\
H_{3}=\frac{2}{\Delta t_{\mathrm{AE}}^{2}} m_{S}-\frac{1}{3} k_{S}^{*} \\
H_{4}=\frac{-1}{\Delta t_{\mathrm{AE}}^{2}} m_{S}-\frac{1}{3} k_{S}^{*}
\end{gathered}
$$

The solution vector at the next time step $d^{(n+1)}$ is obtained by decomposing $H_{1}$ and applying it to the right-hand side of Eq. (13).

\section{Efficient Updating of Stiffness and Thermal Loads}

As the temperature distribution of the structure is continuously changing in time, the stiffness matrix and thermal load vector must be updated during the course of the aerothermoelastic simulation due to their dependence on temperature. Note that, in the current framework, these quantities are updated at every iteration of the structural dynamic response solution. Further investigation is required to develop methodologies for determining the appropriate time interval at which to update the structural stiffness and thermal loads based on the rate of evolution of the thermal solution. Calculation of the generalized stiffness involves generating the physical stiffness matrix and pre- and postmultiplying by the modal matrix. Updating the physical stiffness matrix requires assembling $K_{S}(T)$ based on the temperature dependence of material properties as well as solving a static finite element problem to generate $K_{G}(T)$. Computation of the generalized thermal load vector requires updating the physical thermal load vector and premultiplying by the modal matrix. Creation of the physical thermal load vector requires updating each element load vector based on the temperature change and assembling the global load vector.

Because the number of physical degrees of freedom in the structural model is large, reassembling the physical stiffness matrix and thermal load vector at every aeroelastic time step is undesirable. One goal of this paper is to examine techniques for reducing the computational cost of the structural ROM by avoiding the need to reassemble the physical stiffness matrix and thermal load vector during the course of the aerothermoelastic simulation. A method for directly updating the stiffness matrix and thermal loads based on a given temperature distribution has therefore been developed to reduce the computational cost of the structural ROM. This can be seen as replacing some of the blocks in Fig. 1 with a reduced-order representation linking the transient thermal solution to the structural dynamic equations of motion. The generalized load vector due to aerodynamic loads $f_{S}^{A}$ is still assembled in the usual manner, as it only contains contributions to the loads at the outer surface of the structure and the associated computational cost is relatively low.

\section{A. Overview of Kriging Theory}

The methodology employed in this work is based on the kriging technique [18], which provides a global approximation to a function based on sampled training data. Consider a multi-input/single-output function $y\left(v_{1}, v_{2}, \ldots, v_{n_{\mathrm{iv}}}\right)$, where $y$ represents the scalar output of the function, $v$ denotes the input variables to the function, and $n_{\mathrm{iv}}$ is the number of input variables. Kriging provides an approximation $\hat{y}(b)$ to the function based on local deviations $Z(b, X)$ from a global approximation $R(b, X)$ of the form [19]

$$
\hat{y}(b)=R(b, X)+Z(b, X)
$$

where $b$ is a vector of inputs corresponding to the untried location in the parameter space, and $X$ is a collection of the sample points used for the training data. The training responses at the sample points are stored in the response vector $Y(X)$ such that

$$
Y(X)=\left\{\begin{array}{c}
y^{(1)}\left(X^{(1)}\right) \\
y^{(2)}\left(X^{(2)}\right) \\
\vdots \\
y^{\left(n_{k}\right)}\left(X^{\left(n_{k}\right)}\right)
\end{array}\right\}
$$

where $y^{(j)}\left(X^{(j)}\right)$ indicates the function output for the $j$ th kriging snapshot $X^{(j)}$, and $n_{k}$ is the number of kriging snapshots.

The regression model $R(b, X)$ is an assumed function (usually of polynomial form), whereas $Z(b, X)$ ensures that the kriging model interpolates the sampled data points exactly and is a realization of a stochastic process with zero mean, a variance of $\sigma^{2}$, and a nonzero covariance. Alternatively, $Z(b, X)$ represents uncertainty in the mean of $y(b)$; and the covariance matrix of $Z(b, X)$ for two points in the parameter space, $b^{(i)}$ and $b^{(j)}$, is given by

$$
\operatorname{Cov}\left[Z\left(b^{(i)}\right), Z\left(b^{(j)}\right)\right]=\sigma^{2} C\left[G\left(X^{(i)}, X^{(j)}\right)\right]
$$

where $C$ is the correlation matrix that is assembled based on the chosen correlation function $G\left(X^{(i)}, X^{(j)}\right)$, and $X^{(i)}$ and $X^{(j)}$ are the $i$ th and $j$ th sample points, respectively. In Eq. (17), the process variance $\sigma^{2}$ functions as a scale factor that can be tuned to the training data. The role of the correlation function is to account for the effect of each interpolation point on every other interpolation point and quantifies how quickly and smoothly the function moves from point $X^{(i)}$ to point $X^{(j)}$. In this work, a Gaussian correlation function is used, and it is given by [19]

$$
G\left(X^{(i)}, X^{(j)}\right)=\exp \left[-\sum_{k=1}^{n_{\mathrm{iv}}} \theta_{k}\left|X_{k}^{(i)}-X_{k}^{(j)}\right|^{p_{k}}\right]
$$

where $\theta_{k}$ and $p_{k}$ are the unknown fitting parameters, and $X_{k}^{(i)}$ denotes the $k$ th component of the $i$ th sample point. The bounds on the fitting parameters are $\theta_{k}>0$ and $0<p_{k} \leq 2$. As the point $X^{(i)}$ approaches $X^{(j)}$, Eq. (18) approaches its maximum value of one, leading to the property that the kriging surface passes through the sampled data points. Therefore, the Gaussian correlation function is intuitive in that the closer two points become in the parameter space, the greater the correlation between the two points becomes. The parameters $\theta_{k}$ in Eq. (18) serve to provide a measure of activity in the variable $X_{k}$. Large values of $\theta$ indicate that there is strong correlation only for sample points that are close together. Small values of $\theta$ indicate that sample points spaced further apart still have a strong influence on each other because they are well correlated. An alternative interpretation of the $\theta_{k}$ parameters is associated with dependence of 
the function on the $k$ th input variable. If the function of interest has a strong dependence on the $k$ th input variable, there exist large differences in the function values at $X_{k}^{(i)}$ and $X_{k}^{(j)}$. Thus, the corresponding $\theta_{k}$ parameter will be large such that, even though the absolute difference between $X_{k}^{(i)}$ and $X_{k}^{(j)}$ is small, the correlation between the function values at the $i$ th and $j$ th snapshots is low due to the fact that it changes rapidly with changes in the $k$ th input variable. The exponents $p_{k}$ are related to the smoothness of the function of interest in the direction of the $k$ th input variable, with increasing $p_{k}$ corresponding to increasing smoothness. To determine the fitting parameters, the form of $R(b, x)$ must first be chosen.

To derive the prediction equations, a vector containing the regression functions, denoted by $r_{x}$, is assembled such that

$$
R(b, X)=r_{x}(b)^{T} \beta(X)
$$

where $r_{x}^{T}$ is a $1 \times n_{b}$ row vector of basis functions, with $n_{b}$ being the number of basis functions associated with the regression polynomial; and $\beta$ is an $n_{b} \times 1$ column vector of coefficients of each of the polynomial terms. The expanded design matrix $R_{x}$ is of the form [20]

$$
R_{x}(X)=\left[\begin{array}{c}
r_{x}^{T}\left(X^{(1)}\right) \\
r_{x}^{T}\left(X^{(2)}\right) \\
\vdots \\
r_{x}^{T}\left(X^{\left(n_{k}\right)}\right)
\end{array}\right]
$$

such that the $i$ th row of $R_{x}$ corresponds to the evaluation of the $n_{b}$ basis functions at the $i$ th kriging snapshot. If the stochastic process evaluated at the kriging snapshot points is denoted as

$$
z(X)=\left[Z\left(X^{(1)}\right), Z\left(X^{(2)}\right), \ldots, Z\left(X^{\left(n_{k}\right)}\right)\right]^{T}
$$

then the training data can be represented as

$$
Y(X)=R_{x} \beta+z
$$

The goal of the kriging methodology is to obtain the best linear unbiased predictor, where unbiasedness refers to the fact that the expected value of the predictor must be equal to the expected value of Eq. (22). This is accomplished by solving an optimization problem to minimize the error of the predictor subjected to constraints that ensure unbiasedness. This procedure results in the kriging predictor being given by

$$
\hat{y}(b)=r_{x}^{T} \hat{\beta}+g^{T}(b, X) G^{-1}\left(Y(X)-R_{x} \hat{\beta}\right)
$$

where $Y(X)$ is the column vector of length $n_{k}$ containing the values of the function outputs at the sample points, and $g^{T}(b, X)$ is a correlation vector between the untried point $b$ and the sample data points $X$. Note that Eq. (23) is the equation that is used to give the kriging prediction within the aerothermoelastic framework. The correlation vector $g$ is given by

$$
g(b, X)=\left[G\left(b, X^{(1)}\right), G\left(b, X^{(2)}\right), \ldots, G\left(b, X^{\left(n_{k}\right)}\right)\right]^{T}
$$

In Eq. (23), $\hat{\beta}$ is the generalized least-squares estimator of $\beta$ and is given by

$$
\hat{\beta}=\left(R_{x}^{T} C^{-1} R_{x}\right)^{-1} R_{x}^{T} C^{-1} Y(X)
$$

At this point, one must determine the fitting parameters $\theta_{k}$ and $p_{k}$ in Eq. (18). As the stochastic process associated with the error of the kriging regressors is assumed to be Gaussian, the optimal values of $\theta_{k}$ and $p_{k}$ are those that maximize the likelihood that the interpolation points have been drawn from such a process. Alternatively stated, we seek to choose $\theta_{k}$ and $p_{k}$ leading to a kriging function such that the consistency between the actual model and the kriging predictions of the model is maximized. The problem of obtaining the maximum likelihood estimates of $\theta_{k}$ and $p_{k}$ is posed as

$$
\min _{\theta_{k}>0,0<p_{k} \leq 2}-\frac{n_{k} \ln \left(\hat{\sigma}^{2}\right)+\ln |C|}{2}
$$

where $|C|$ is the determinant of $C$, and $\hat{\sigma}$ is the generalized leastsquares estimate of $\sigma$ given by

$$
\hat{\sigma}^{2}=\frac{\left(y-R_{x} \hat{\beta}\right)^{T} C^{-1}\left(y-R_{x} \hat{\beta}\right)}{n_{k}}
$$

The maximum likelihood estimate given in Eq. (26) is a function of the $\theta_{k}$ and $p_{k}$ parameters only, and one can thus use nonlinear optimization techniques to obtain these parameters. Although any values of $\theta_{k}$ and $p_{k}$ would result in a kriging model that interpolates the sample points exactly, the "best" kriging model is that which uses those values of $\theta_{k}$ and $p_{k}$ that minimize the function given in Eq. (26).

In this work, kriging is used to approximate vector and matrix quantities. Therefore, an independent kriging predictor is generated for each entry in the corresponding vector or matrix. The training responses at the sample points are stored in matrix form such that $Y(X)$ becomes

$$
Y(X)=\left[\begin{array}{cccc}
y_{1}^{(1)}\left(X^{(1)}\right) & y_{2}^{(1)}\left(X^{(1)}\right) & \cdots & y_{n_{p}}^{(1)}\left(X^{(1)}\right) \\
y_{1}^{(2)}\left(X^{(2)}\right) & y_{2}^{(2)}\left(X^{(2)}\right) & \cdots & y_{n_{p}}^{(2)}\left(X^{(2)}\right) \\
\vdots & \vdots & \ddots & \vdots \\
y_{1}^{\left(n_{k}\right)}\left(X^{\left(n_{k}\right)}\right) & y_{2}^{(2)}\left(X^{\left(n_{k}\right)}\right) & \cdots & y_{n_{p}}^{\left(n_{k}\right)}\left(X^{\left(n_{k}\right)}\right)
\end{array}\right]
$$

where $y_{i}^{(j)}$ indicates the $i$ th entry of the response vector for the $j$ th kriging snapshot $X^{(j)}$, and $n_{p}$ is the number of output parameters in a snapshot. Thus, a kriging predictor is generated for each column of $Y(X)$ as shown in Eq. (28).

\section{B. Use of Kriging for Efficient Updating of Stiffness and Thermal} Loads

In this application, the quantities to be approximated using kriging $\hat{y}$ are the entries of the stiffness matrix and the thermal load vector, and the inputs to function $b$ are spatially varying temperature distributions. Because the current full-order thermal model contains thousands of degrees of freedom, it is impractical to treat each thermal degree of freedom as a variable in the parameter space. This is due to the fact that the resulting parameter space would be too large to realistically sample when generating the kriging training data. However, as POD is already being used for the thermal ROM, it provides a convenient and optimal means for parameterizing the transient temperature distribution in terms of a small number of variables. By using the truncated set of thermal POD modal coordinates as the parameters to represent the complete temperature distribution, the number of parameters to be sampled is greatly reduced. The large-scale reduction in the number of input variables needed to represent the physical temperature distribution is due to the optimality of the POD basis. Recall that the POD basis is optimal in the sense that it captures the solution more accurately in a finite number of modes than any other basis representation using the same number of modes, provided that the POD snapshots adequately capture the dynamics of the system. This optimality is critical as the computational effort required to construct a kriging model is a strong function of the number of input variables involved.

Because of the large number of structural degrees of freedom, the computational cost and memory associated with updating the physical stiffness matrix $K_{S}^{*}(T)$ and pre- and postmultiplying by the structural reference modes at every aeroelastic time step is undesirable. As such, kriging is used to directly create the generalized stiffness matrix $k_{S}^{*}(T)$, which is of reduced size and does not possess the computational issues associated with the physical stiffness matrix. As the number of entries in the physical thermal load vector $F_{S}^{H}$ is reasonable, error analysis is conducted on kriging models for both the physical and generalized thermal load vectors to assess which one can be more accurately represented. 


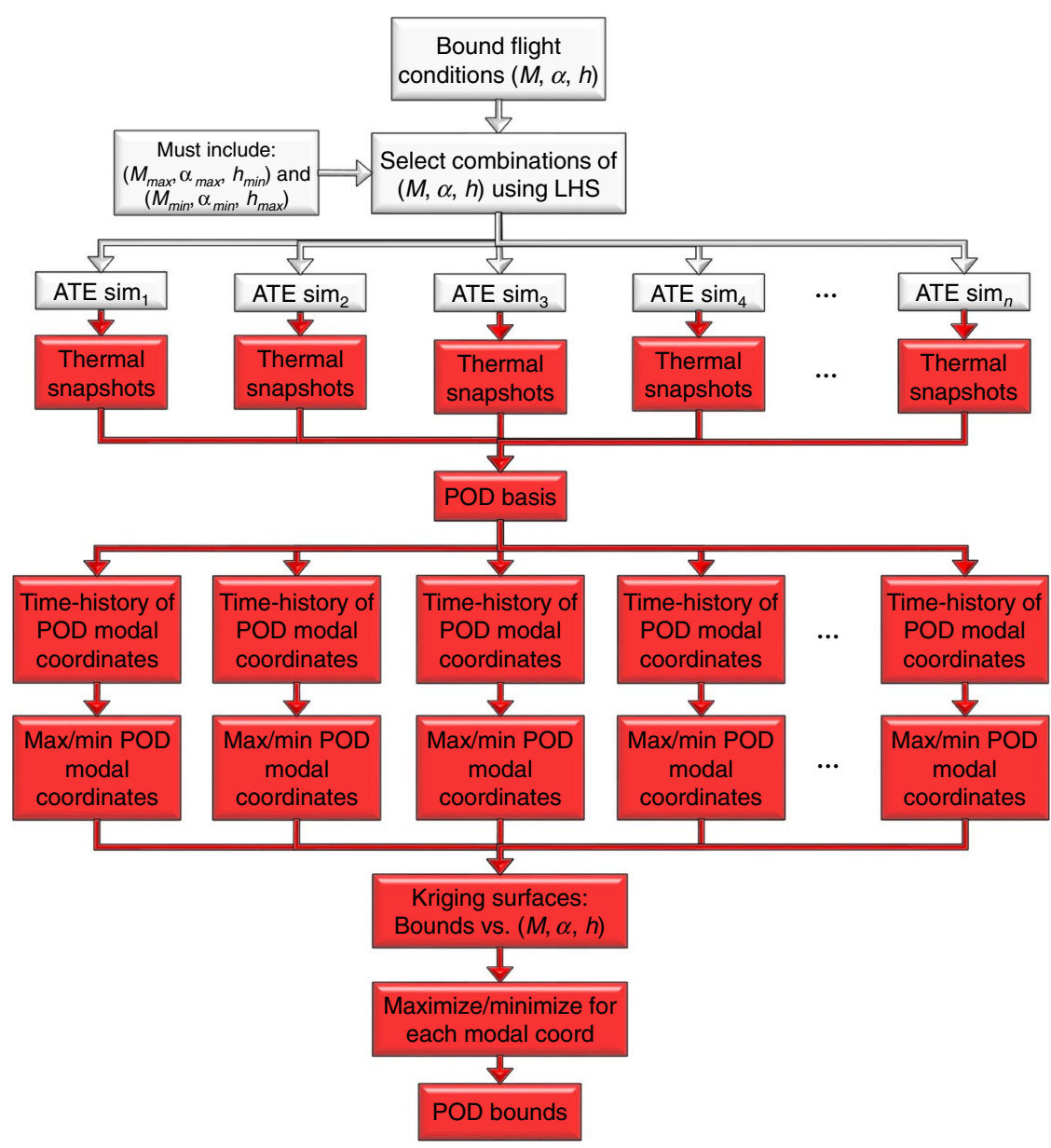

Fig. 4 Proposed methodology for generating thermal snapshots and bounding POD modal coordinates.

\section{Methodology for a Priori Bounding of POD Modal Coordinates}

It is important to establish a robust methodology for bounding the POD modal coordinates a priori, as there is no guarantee of accuracy if they go outside of the bounds that were used in generating the kriging training data. As such, a robust and efficient method for bounding the POD modal coordinates is developed. An overview of the proposed methodology is given in Fig. 4.

The process begins by identifying the range of flight conditions for which the kriging ROMs are to be valid. The flight conditions of interest are the Mach number, angle of attack, and altitude. Using the bounds on the flight conditions, a Latin hypercube sampling (LHS) procedure is used to generate $n$ flight condition sets. One of the sample points must correspond to the highest dynamic pressure case (maximum Mach number, maximum angle of attack, and minimum altitude), and another sample point must correspond to the lowest dynamic pressure case (minimum Mach number, minimum angle of attack, and maximum altitude). For each set of flight conditions, a full-order aerothermoelastic simulation is carried out and thermal snapshots are taken. Note that these simulations are performed in parallel such that the time to generate all of the thermal snapshots is the computation time for a single full-order simulation. Once the thermal snapshots are obtained from all of the full-order aerothermoelastic simulations, they are combined into a single snapshot matrix and the POD basis is generated. The POD basis is then used along with the snapshots to calculate what the time history of the POD modal coordinates would have been had the thermal ROM been used for each set of flight conditions. For each case, the maximum and minimum values of each POD modal coordinate are identified, and this information is used to create a kriging surface that gives the bounds on each of the POD modal coordinates as a function of the Mach number, angle of attack, and altitude. This kriging surface is then used as a surrogate model for the purpose of finding the maximum possible upper bound and minimum possible lower bound for each POD modal coordinate at any location within the parameter space of the flight conditions. Once the POD bounds are established, they are used in the generation of sample points for the kriging ROMs of the stiffness matrix and thermal load vector. This framework serves two important purposes. In addition to providing robust bounds on the POD modal coordinates, it also leads to the generation of a rich set of thermal snapshots, leading to an accurate thermal POD model.

One challenge involved in bounding the POD modal coordinates is associated with their initial conditions. If the relations given in Eq. (4) are solved directly for the POD modal coordinates, it is likely that the bounds will be exceeded for simulations for which the initial conditions vary from those at which the bounds were generated. It is therefore desirable to generate bounds that are independent of the initial conditions by transforming Eq. (3) such that the initial conditions are made to be uniform and homogeneous. This is accomplished by defining a new variable $\tilde{T}$, which represents the excess of temperature over the initial condition, i.e.,

$$
\tilde{T}(t)=T(t)-T_{0}
$$

where $T_{0}$ is the vector of initial temperatures. Applying this transformation to Eq. (3), the system becomes

$$
M \dot{\tilde{T}}(t)+K \tilde{T}(t)=G_{T}(t)
$$

where $G_{T}(t)=F(t)-K T_{0}$. As before, the system is transformed to modal space using

$$
\bar{\Phi}_{T}^{T} M_{T} \bar{\Phi}_{T} \dot{\tilde{c}}(t)+\bar{\Phi}_{T}^{T} K_{T} \bar{\Phi}_{T} \tilde{c}(t)=\bar{\Phi}_{T}^{T} G_{T}(t)
$$

where $\tilde{c}$ denotes the fact that the system has been transformed to impose homogeneous initial conditions. Once the system is solved 
for $\tilde{c}(t)$, the physical temperatures are obtained by multiplying the POD basis with the POD modal coordinates and adding the initial conditions back on, i.e.,

$$
T(t)=\bar{\Phi} \tilde{c}(t)+T_{0}
$$

In addition to making the bounds independent of the initial conditions, this transformation provides another benefit in terms of improving the accuracy of the thermal ROM. If the initial conditions were not made to be homogeneous, the initial value of each POD modal coordinate would be found by projecting $T_{0}$ in the direction of the corresponding POD basis vector, i.e.,

$$
\boldsymbol{c}_{i}(0)=\left\langle\varphi^{(i)}, T_{0}\right\rangle
$$

where $\langle\cdot\rangle$ denotes an inner product. Because the POD basis does not form a complete space for the physical temperatures, the initial temperature distribution cannot be represented exactly in this formulation. However, by transforming the equations to enforce homogeneous initial conditions, $\tilde{c}(0)$ is forced to become a vector of zeros and $T(t)$ can be represented exactly after the vector of initial conditions is added back to the solution. For the current framework in which Eq. (31) is integrated numerically and the solution at each time step depends on that at the previous time step, the ability to represent the initial conditions exactly is important.

To account for the transformation of Eq. (29) in the kriging representation of the stiffness matrix and thermal loads, additional input variables corresponding to $T_{0}$ must be included in the kriging functions. This is necessary because the input variables passed to the kriging functions must be capable of reproducing the physical temperature distribution, including the effect of the initial condition. In this work, the initial temperature distribution is assumed to always be uniform such that the physical temperature distribution can be expressed as

$$
T(t)=\left[\sum_{i=1}^{r} c_{i}(t) \varphi^{(i)}\right]+\mathcal{T}_{0}\{1\}
$$

where $\mathcal{T}_{0}$ is the scalar value of the uniform initial temperature, and $\{1\}$ is a vector of ones of length $s$. Thus, the input variables for the kriging ROMS are the POD modal coordinates $\boldsymbol{c}_{i}$ and the scalar value of the initial temperature $\mathcal{T}_{0}$. Because the initial condition is taken to be uniform here, only one additional input variable is required to represent the initial condition. Arbitrary spatial variation of the initial conditions can be permitted by replacing $\mathcal{T}_{0}\{1\}$ in the right-hand side of Eq. (34) with a linear combination of multiple basis vectors. Note that this would require a larger number of input variables to represent the initial conditions.

\section{Selection of Parameters for Kriging Model Generation}

The number of input variables, number of sample points, and order of regression must be selected for both the kriging model of the thermal loads and the kriging model of the generalized stiffness. In selecting these parameters, consideration must be given to a variety of factors. The number of sample points used to generate the kriging ROMs must be sufficient to provide enough training information to achieve the desired level of accuracy. Additionally, if too few input variables are used, the temperature distribution passed to the kriging model may not be representative of the actual temperature distribution. Finally, the computation time required to generate the kriging ROMs must be balanced against the desired accuracy, number of sample points, and number of input variables required to obtain this accuracy for a given regression model. Because the computation time increases quickly with number of sample points, number of input variables, and order of regression, the tradeoff between desired accuracy and computation time must be taken into account. Though the kriging models are generated a priori and offline, the computation time required to generate them must still be reasonable.

\section{Techniques for Enhanced Modal Solutions}

Previous work [13] has shown that the use of free vibration modes alone within a mode-displacement approach for reduced-order structural dynamic modeling may not always be sufficient to capture the structural dynamic response under the aerodynamic and thermal loads experienced in hypersonic flight. Thus, the goal of the current section is to outline techniques for improving the modal representation of the structural dynamic response while still maintaining the low-order nature of the solution. Because both the thermal and structural ROMs consist of modal solutions, the techniques discussed are applicable to both. However, in this paper, the techniques are applied only to the structural dynamic model.

The technique used in this study to improve the accuracy of the structural ROM involves augmentation of the original basis with additional Ritz vectors. In this approach, the original structural basis containing the reference free vibration modes is augmented by inserting additional columns into the modal matrix, i.e.,

$$
\Phi_{S}=\left[\begin{array}{ccccccc}
\phi_{1}^{(1)} & \phi_{1}^{(2)} & \cdots & \phi_{1}^{(r)} & \psi_{1}^{(1)} & \cdots & \psi_{1}^{(l)} \\
\phi_{2}^{(1)} & \phi_{2}^{(2)} & \cdots & \phi_{2}^{(r)} & \psi_{2}^{(1)} & \cdots & \psi_{2}^{(l)} \\
\vdots & \vdots & \ddots & \vdots & \vdots & \ddots & \vdots \\
\phi_{s}^{(1)} & \phi_{s}^{(2)} & \cdots & \phi_{s}^{(r)} & \psi_{s}^{(1)} & \cdots & \psi_{s}^{(l)}
\end{array}\right]
$$

where $\psi^{(i)}$ represents the $i$ th augmented mode shape, and $l$ is the number of augmented modes. The specific technique used in this work to obtain the augmented mode shapes is the method of loaddependent Ritz vectors [21]. This approach is advantageous in that it provides the capability for capturing quasi-static structural response that might otherwise not be contained within a particular eigenvector subspace. Thus, load-dependent Ritz vectors provide a means for incorporating the spatial distribution of the loads into the modal representation of the structural dynamics.

A summary of the algorithm for computing load-dependent Ritz vectors is given in Algorithm 1 . The procedure begins by selecting a set of free vibration modes $\phi$. In this application, the number of free vibration modes $r$ is determined based on a cutoff frequency beyond which the corresponding mode shapes are not expected to contribute to the solution. The physical mass matrix $M_{S}$ and modified stiffness matrix at a reference thermal state $K_{S}^{*}$ are then obtained from the finite element model, and $K_{S}^{*}$ is decomposed into its lower-upper (LU) factorization for efficient inversion. The first load-dependent Ritz vector $\bar{\psi}^{(1)}$ is computed in step 4 by solving the static problem given by

$$
K_{S}^{*} \bar{\psi}^{(1)}=F_{S}^{I}
$$

where $F_{S}^{I}$ is a user-determined input. The quality of the load-dependent Ritz vectors is dependent on the degree to which $F_{S}^{I}$ represents the loading that the structure will experience in the actual simulation. To make $\bar{\psi}^{(1)}$ orthogonal to the previously determined free vibration modes with respect to $M_{S}$, the modified Gram-Schmidt algorithm is invoked in step 5 , which repeatedly removes from $\bar{\psi}^{(1)}$ its projection onto the previous modes according to

$$
\bar{\psi}^{(1)}=\bar{\psi}^{(1)}-\phi^{(j)} \phi^{(j) T} M_{S} \bar{\psi}^{(1)}, \quad j=1, \ldots, r
$$

In Eq. (37), every time $\bar{\psi}^{(1)}$ is updated, it overwrites the previous version, thus reducing storage requirements. Note that the modified Gram-Schmidt algorithm is used in order to avoid the well-known numerical instabilities associated with the classical Gram-Schmidt algorithm. The orthogonalized solution vector $\bar{\psi}^{(1)}$ is then normalized with respect to the mass matrix to obtain $\psi^{(1)}$ using

$$
\psi^{(1)}=\frac{\bar{\psi}^{(1)}}{\left(\bar{\psi}^{(1) T} M_{S} \bar{\psi}^{(1)}\right)^{1 / 2}}
$$

The static solution used to obtain $\psi^{(1)}$ neglected the inertial forces, which are given by $M_{S} \ddot{\psi}^{(1)}$. Assuming harmonic motion in free 
Algorithm 1 Algorithm for generation of load-dependent Ritz vectors [21]

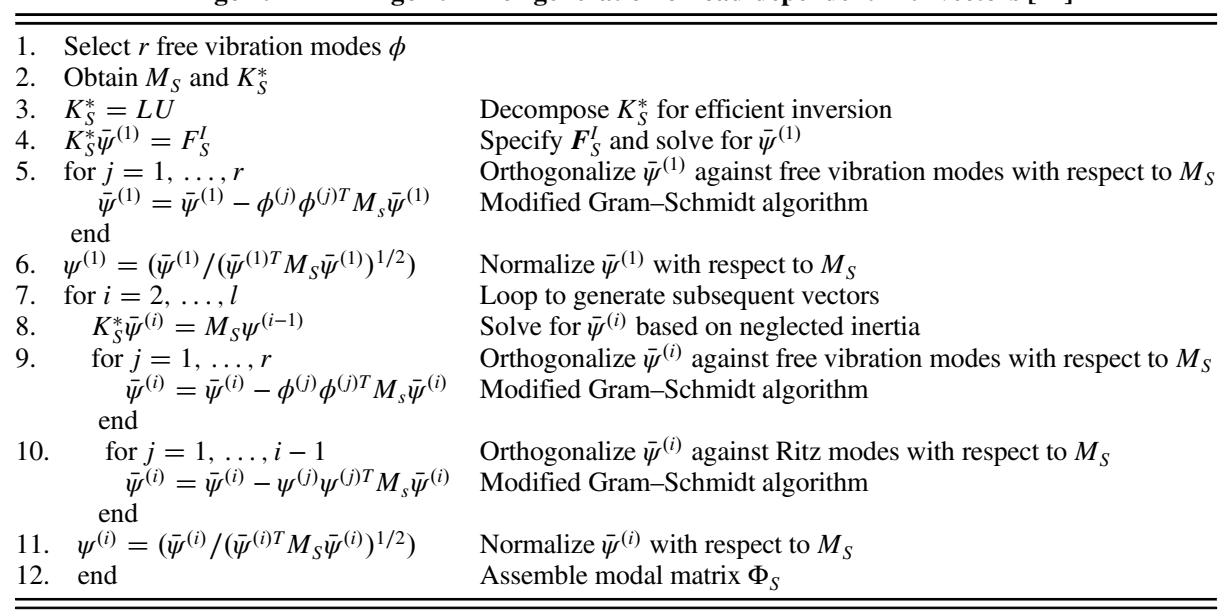

vibration, the neglected inertial forces are of the form $\omega^{2} M_{S} \psi^{(1)}$, where $\omega$ represents a typical frequency of the load. The vector of neglected inertial loads is then applied as a load vector in the generation of $\bar{\psi}^{(2)}$ at step 8 . Again, a static solution is used to obtain $\bar{\psi}^{(2)}$, and $\psi^{(2)}$ is obtained by orthogonalizing in mass against all other modes and normalizing with respect to the mass matrix. Steps 8-11 are repeated until the desired number of load-dependent Ritz vectors are obtained. The procedure depicted in Algorithm 1 shows the steps taken when a single load vector $F_{S}^{I}$ is specified. The algorithm can be generalized for the case in which multiple load vectors are specified. In that case, steps 4-11 are repeated for each $F_{S}^{I}$; each time a new load-dependent Ritz vector $\bar{\psi}$ is calculated, it is orthogonalized against all previous vectors with respect to $M_{S}$, and it is normalized with respect to $M_{S}$. The number of specified load vectors $n_{F}$ and number of load-dependent Ritz vectors per specified load vector $n_{R}$ will affect the solution accuracy. Note that $n_{R}$ corresponds to the number of iterations performed in steps $7-11$.

The main challenge associated with this approach is determining the representative load vectors $F_{S}^{I}$ to use in step 4 of Algorithm 1 . As the quality of the basis is dependent on the choice of $F_{S}^{I}$, it is important to choose these vectors so that they most closely represent the spatial distribution of loads that the structure will experience during flight. To identify the dominant spatial components of the loads, a POD analysis is applied to the structural load vector $F_{S}$ based on snapshots of $F_{S}$ from high-fidelity aerothermoelastic simulations. As discussed in Sec. V.A, the POD is optimal in the sense that it arranges the modes such that the first mode contains the largest amount of energy, the second mode contains the second largest amount of energy, and so on. As a result of this optimality property, POD can be used to identify the most dominant spatial components of the structural loads for use in the load-dependent Ritz vector procedure. Algorithm 1 will be repeated for a specified number of representative load vectors, resulting in a set of load-dependent Ritz vectors to be appended to the set of free vibration modes.

\section{A. Lifting Surface Model}

\section{Numerical Studies}

A finite element model of a representative hypersonic vehicle allmovable lifting surface has been created for use in this study. A detailed description of the structure was provided in [13], and thus only a brief overview is given here. The thickness from the top skin layer to the bottom skin layer is $4 \%$ chord length [22]. The top and bottom skin layers are each equipped with two 3.8-mm-thick thermal protection system layers; thus, the thickness of the outer mold line is $4 \%$ chord length plus the $15.2 \mathrm{~mm}$ of thermal protection system material. The chord length at the root is $5.2 \mathrm{~m}$. (17 ft) [23], and the leading edge makes an angle of $34 \mathrm{deg}$ with the $y$ axis, whereas the trailing edge makes an angle of $18 \mathrm{deg}$ with the $y$ axis [24]. Planform and cross-sectional views of the lifting surface are given in Figs. $\underline{5}$ and $\underline{6}$, respectively.
This study considers a thermal protection system consisting of an outer heat shield and middle insulation layer on top of the skin, as shown in Fig. 7. The material for the heat shield is chosen to be René 41 , as it was found to be efficient in terms of mechanical properties at elevated temperatures. Min-K insulation, which is a proprietary silica-based material faced with Astroquartz ${ }^{\circledR}$ cloth [25], is selected for the insulation layer due to its relatively low thermal diffusivity. For the structure (both skin and stiffeners), the Titanium alloy TIMETAL 834 (formerly known as IMI 834) is chosen.

The thermal and mechanical properties of the three materials employed in the model are shown in Table 1, where "T-dep." indicates that the property is temperature dependent [25-27]. Note that the temperature-dependent material properties for René 41 are extrapolated beyond the available data, and the maximum application temperature $T_{\max }$ is set to $1500 \mathrm{~K}$. The emissivity of the heat shield is taken to be 0.85 [24]. The thermal strain is calculated based on the temperature change with respect to a reference stress-free

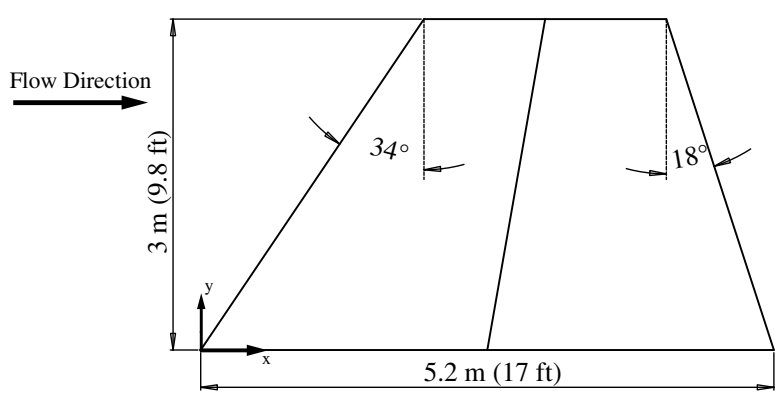

Fig. 5 Planform geometry of lifting surface model.

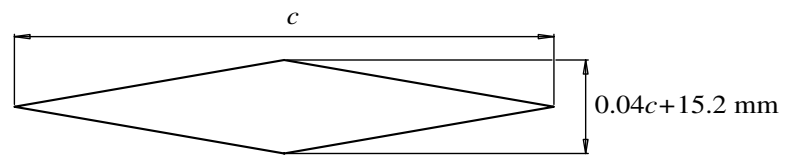

Fig. 6 Cross-sectional geometry of lifting surface model.

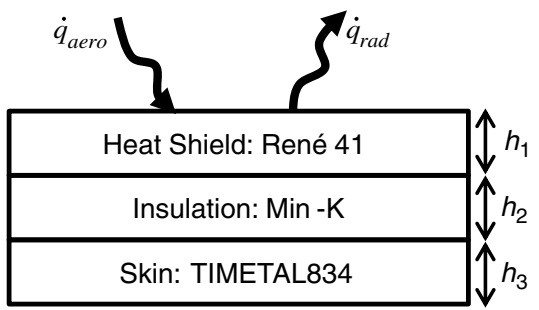

Fig. 7 Schematic of material stacking scheme at outer mold line of structure. 
Table 1 Structural and thermal material properties used in the study

\begin{tabular}{lcccccccc}
\hline \hline & $\rho, \mathrm{kg} / \mathrm{m}^{3}$ & $E, \mathrm{~Pa}$ & $\nu$ & $\alpha_{T}, \mu \mathrm{m} / \mathrm{m} / \mathrm{K}$ & $\kappa, \mathrm{W} / \mathrm{m} / \mathrm{K}$ & $\boldsymbol{c}_{p}, \mathrm{~J} / \mathrm{kg} / \mathrm{K}$ & $T_{\max }, \mathrm{K}$ & $h, \mathrm{~mm}$ \\
\hline Heat shield & 8240 & T-dep. & 0.31 & T-dep. & 18 & 541 & 1500 & 3.8 \\
Insulation & 256 & Neglect & Neglect & Neglect & 0.052 & 858 & 1250 & 3.8 \\
Skin & 4550 & T-dep. & 0.31 & 11 & 7 & 525 & 873 & 6.35 \\
\hline \hline
\end{tabular}

The properties $\nu, \kappa$, and $\boldsymbol{c}_{p}$ for TIMETAL 834 were obtained from http://www.matweb.com/search/DataSheet.aspx?

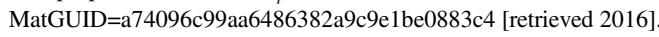

temperature. The reference temperature used for calculation of thermal strain is taken to be $293 \mathrm{~K}$ for all materials. The finite element model used for the thermal and structural modeling aspects of the study as described in [13] is shown in Fig. 8 with the top surface removed for visualization purposes.

\section{B. Bounding the POD Modal Coordinates}

To demonstrate the previously outlined approach for bounding the POD modal coordinates, the framework of Fig. 4 is applied to the lifting surface structure described previously for the ranges in flight conditions shown in Table 2. Ten sets of flight conditions are identified using LHS. Two different sets of time-step sizes are used in the aerothermoelastic simulations. For the period of $0-150 \mathrm{~s}$, the time-step sizes are $\Delta t_{\mathrm{HT}}=\Delta t_{\mathrm{AE}}=0.1 \mathrm{~s}$ and $\Delta t_{\mathrm{AT}}=1 \mathrm{~s}$. For the period from 150 to $3600 \mathrm{~s}$, the time-step sizes are $\Delta t_{\mathrm{HT}}=\Delta t_{\mathrm{AE}}=$ $1 \mathrm{~s}$ and $\Delta t_{\mathrm{AT}}=5 \mathrm{~s}$. One thermal snapshot is taken at each heat transfer time step. This results in total of 4951 snapshots for each of the 10 full-order aerothermoelastic simulations, and thus a total of 49,510 snapshots are used in the generation of the POD basis.

Using the 49,510 snapshots obtained from the 10 full-order aerothermoelastic simulations, the POD basis is generated. The eigenvalues associated with the first 50 POD modes are given in Fig. 9. Based on the eigenvalues, the first 32 POD vectors are retained after basis truncation in this case. Though the thermal ROM is not the focus of this paper, its accuracy is assessed for one set of flight conditions. The flight conditions used for verifying the accuracy of the POD basis are as follows: $M_{\infty}=6.5, \alpha=2 \mathrm{deg}$, and $h=35 \mathrm{~km}$. The time steps for this case are $\Delta t_{\mathrm{AE}}=\Delta t_{\mathrm{HT}}=0.1 \mathrm{~s}$ and $\Delta t_{\mathrm{AT}}=1 \mathrm{~s}$. The normalized root mean-squared error (NRMSE) and $L_{\infty}$ error of the thermal ROM are calculated with respect to the fullorder thermal model at each heat transfer time step using Eqs. (40) and (41). In the case of the thermal ROM, the vectors "Full" and "ROM" correspond to the temperatures vectors from the full-order and reduced-order thermal models at a given time step. To eliminate any additional errors, the full-order structural model is used for both cases. Time histories of the NRMSE and $L_{\infty}$ error for a simulation time of $1200 \mathrm{~s}$ are given in Figs. 10a and 10b, respectively. Examining these figures, the thermal ROM shows good agreement with the fullorder thermal model.

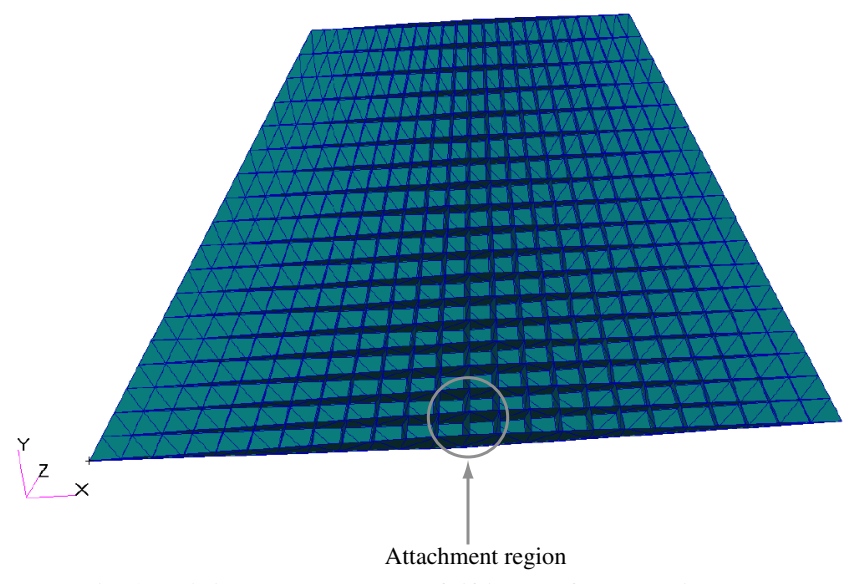

Fig. 8 Finite element model of lifting surface used in study.
Table 2 Bounds on flight conditions for kriging ROM generation

\begin{tabular}{lcc}
\hline \hline Lower bound & Parameter & Upper bound \\
\hline 5.0 & $\leq M_{\infty} \leq$ & 8.0 \\
$0.0 \mathrm{deg}$ & $\leq \alpha \leq$ & $4.0 \mathrm{deg}$ \\
$25.0 \mathrm{~km}$ & $\leq h \leq$ & $45.0 \mathrm{~km}$ \\
$293 \mathrm{~K}$ & $\leq \mathcal{T}_{0} \leq$ & $1500 \mathrm{~K}$ \\
\hline \hline
\end{tabular}

The next step is to obtain the upper and lower bounds for each of the 32 POD modal coordinates using the procedure described in Sec. VI.C. The maximization/minimization procedures are performed using the function fmincon available within MATLAB®. Note that the minimization/maximization steps are repeated at different starting locations within the parameter space in order to avoid the potential for obtaining local extrema. Plots of the upper and lower bounds of each of the 32 POD modal coordinates are given in Figs. $11 \mathrm{a}$ and $11 \mathrm{~b}$, respectively.

To verify that the bounds identified in Fig. 11 are indeed the maximum upper bounds and minimum lower bounds over the range of flight conditions given in Table 2, an additional set of 10 aerothermoelastic simulations is run. The Mach number, angle of attack, and altitude for these simulations are selected by using the LHS methodology along with the criterion to maximize the minimum Euclidean distance between the flight conditions of the 10 new simulations and the 10 simulations that were used in establishing the bounds originally. The additional 10 simulations make use of the thermal ROM that consists of the 32 retained POD modes; however, the full-order structural model is used to calculate the structural dynamic response. The time-step sizes for the additional simulations are the same as those that were used in the original 10 aerothermoelastic simulations. At each heat transfer time step, the vector of POD modal coordinates is stored. The maximum and minimum values of each POD modal coordinate over all time steps over all of the 10 simulations is then found. The bounds identified using the procedure shown in Fig. 4 are compared with the minimum/ maximum values identified via the 10 additional aerothermoelastic simulations by computing margins of safety. The margin of safety for the upper bounds $\mathrm{MS}_{U}$ and margin of safety for the lower bounds $\mathrm{MS}_{L}$ are found using

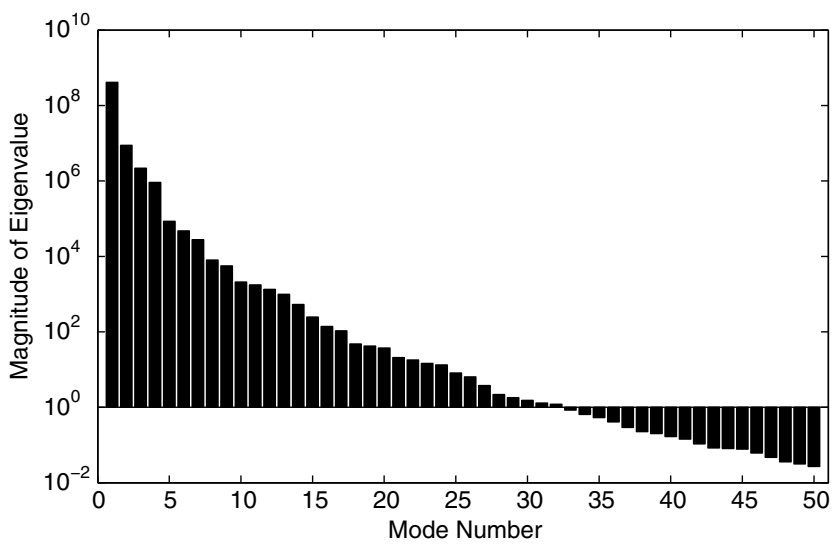

Fig. 9 Eigenvalues associated with first 50 thermal POD modes based on 49,510 thermal snapshots. 

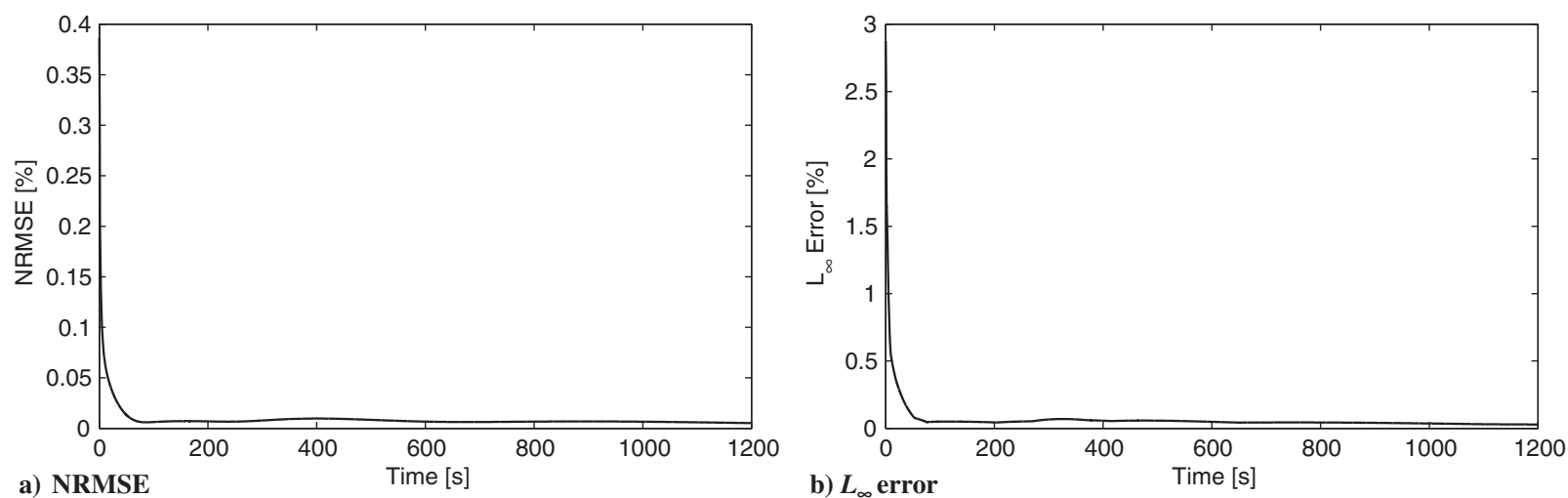

Fig. 10 NRMSE and $L_{\infty}$ error of thermal POD ROM at $M_{\infty}=6.5, \alpha=2 \mathrm{deg}$, and $h=35 \mathrm{~km}$.
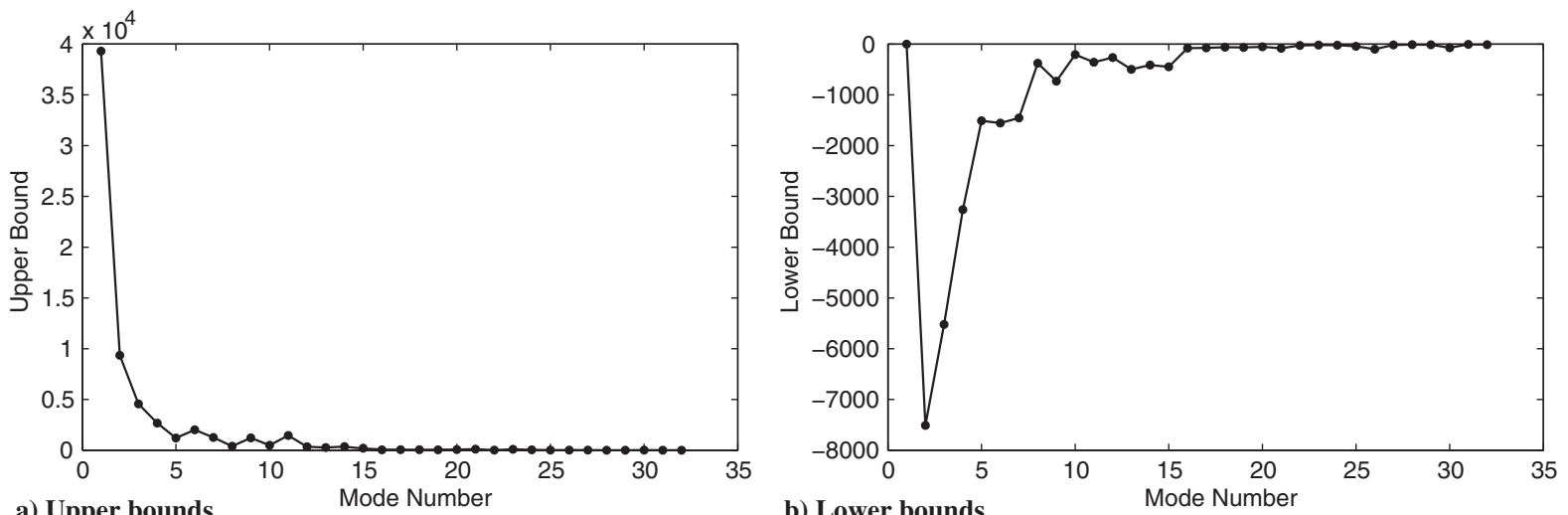

Fig. 11 Upper and lower bounds of POD modal coordinates for 32 retained POD basis vectors.

$$
\begin{aligned}
\mathrm{MS}_{U, i} & =\frac{\mathrm{ub}_{i}-\max _{i}}{\left|\max _{i}\right|} \times 100 \% \\
\mathrm{MS}_{L, i} & =-\frac{\mathrm{lb}_{i}-\min _{i}}{\left|\min _{i}\right|} \times 100 \%
\end{aligned}
$$

where $\mathrm{ub}_{i}$ and $\mathrm{lb}_{i}$ are the upper and lower bounds for the $i$ th POD modal coordinate, and $\max _{i}$ and $\min _{i}$ are the maximum and minimum values of the $i$ th modal coordinate identified from the 10 additional aerothermoelastic simulations. For the upper and lower bounds to encompass the ranges of the POD modal coordinates experienced in the simulations, all elements of both $\mathrm{MS}_{U}$ and $\mathrm{MS}_{L}$ must be greater than or equal to zero. Plots of $\mathrm{MS}_{U}$ and $\mathrm{MS}_{L}$ are given in Figs. 12a and $12 \mathrm{~b}$, respectively. In both figures, all values of the margin of safety are greater than or equal to zero, and thus the bounds are valid. Note that the data point corresponding to mode 1 in Fig. 12b is not shown because both $\min _{1}$ and $l_{1}$ are zero.

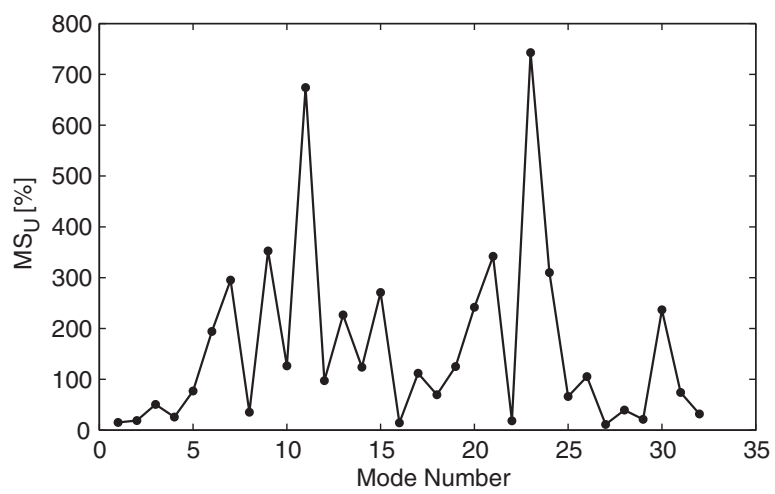

a) Margins of safety on upper bounds
Due to the prohibitive computational expense, it is not feasible to use all 32 POD modal coordinates as input variables in the kriging representations of the stiffness matrix and thermal load vector. Although all 32 POD modes will be used in the solution of the reduced thermal system, only a subset of the resulting POD modal coordinates will be passed to the kriging functions at each aeroelastic time step. Though some accuracy will be lost in representing the physical temperatures using less than 32 POD modes, the penalty is not expected to be significant due to the fact that the POD modes are sorted in order of decreasing energy and the modes that are excluded from the kriging models are chosen to be the lowest energy modes.

\section{Kriging for Efficient Updating of Stiffness and Thermal Loads}

The methodology for efficient updating of stiffness and thermal loads outlined in Sec. VI.B is now demonstrated. The first step in the process is to generate the thermal POD vectors to be used in parameterizing the transient temperature distribution. For the purposes of this section, an aerothermoelastic simulation is run for a

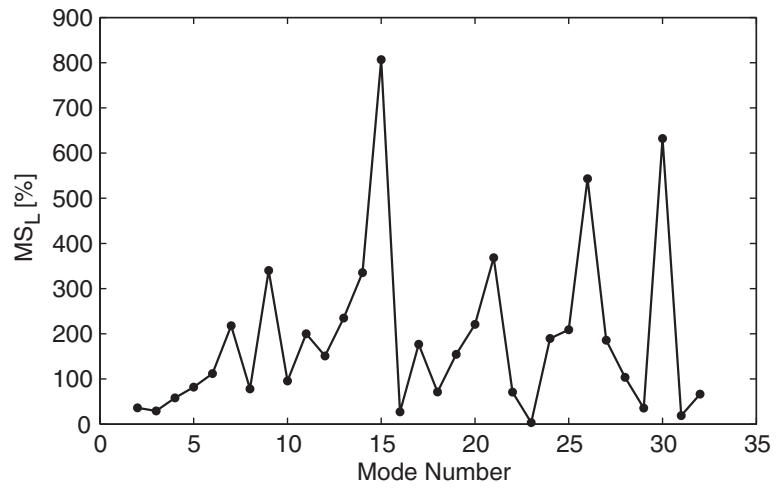

b) Margins of safety on lower bounds

Fig. 12 Margins of safety on upper and lower bounds based on 10 additional aerothermoelastic simulations. 


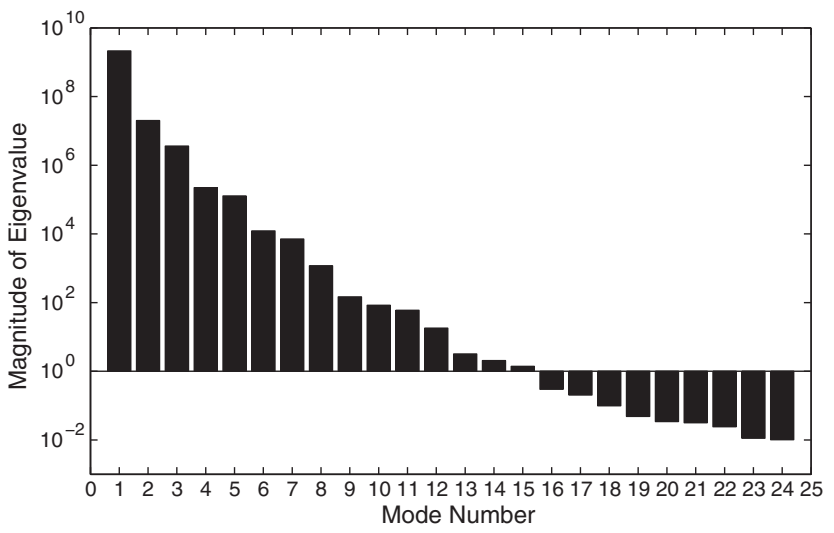

Fig. 13 Semilog plot of first 24 eigenvalues of correlation matrix.

time history of $1 \mathrm{~h}$ at $M=8, h=26 \mathrm{~km}, \alpha=3 \mathrm{deg}$, and a uniform initial temperature distribution $T_{0}$ of $293 \mathrm{~K}$. In a previous work by Falkiewicz et al. [13], it was found that there is a steep initial temperature gradient as the structure begins heating up, and the maximum temperature increases until approximately $150 \mathrm{~s}$ into the transient, at which point it begins to reach a steady state. As such, two different sets of time steps are used to adequately capture the dynamics of the thermal system. For the period from 0 to $150 \mathrm{~s}$, the time steps are chosen as follows: $\Delta t_{\mathrm{HT}}=\Delta t_{\mathrm{AE}}=0.1 \mathrm{~s}$ and $\Delta t_{\mathrm{AT}}=1 \mathrm{~s}$. For the period from 150 to $3600 \mathrm{~s}$, the time steps are chosen as follows: $\Delta t_{\mathrm{HT}}=\Delta t_{\mathrm{AE}}=1 \mathrm{~s}$ and $\Delta t_{\mathrm{AT}}=5 \mathrm{~s}$. The goal of using smaller time steps initially is to provide more resolution for the period in which the dynamics of the thermal system are changing on a faster timescale. One thermal snapshot is taken at each heat transfer time step, regardless of the size of the time step, resulting in a total of 4951 snapshots. The first 24 eigenvalues of the correlation matrix are shown in Fig. 13. Based on the eigenvalues, the first 15 POD modes are retained.

With the input variables chosen, the next step of the process is to generate the kriging sample points to be used in the ROM construction. The LHS methodology is used to generate the kriging sample points. Each kriging sample point consists of a set of 15 POD modal coordinates. For the purposes of the error analysis in this section, the bounds on the POD modal coordinates are computed based on least-squares solutions using the thermal snapshots.

Because the sampling is performed on the POD modal coordinates and not on the nodal temperatures themselves, the resulting temperature vector may be out of the expected range for the structure for certain combinations of POD modal coordinates. To account for this, bounds are also established on minimum and maximum allowable values of the resulting nodal temperatures. After the sample points are generated, the temperature vector corresponding to each sample point is found using the chosen POD basis. A filtering process is then employed that eliminates sample points for which the corresponding nodal temperature values lie outside the range of 293$1500 \mathrm{~K}$. It is therefore necessary to generate more sample points than desired, as not all sample points will be accepted.

Once the inputs for each training run are generated, a high-fidelity analysis is conducted to generate the training data for each sample point. This step consists of generating the temperature distribution corresponding to the selected POD modal coordinates and computing the resulting stiffness matrix and thermal load vector at that temperature distribution. The generalized stiffness matrix calculation is performed by assembling the physical stiffness matrix and pre- and postmultiplying by the chosen structural reference modes. The physical stiffness matrix is computed using Eq. (7) and contains contributions from both the conventional stiffness matrix with temperature-dependent material properties $K_{S}(T)$ and the geometric stiffness resulting from thermal stresses $K_{G}(T)$. The assembling of the matrices and vectors required for the kriging training process is accomplished via the use of Nastran direct matrix abstraction programming. For this case, the structural reference modes are chosen to be the first 10 free vibration modes at the reference thermal state. The reference thermal state is obtained by averaging the 4951 thermal snapshots used in generating the POD basis. Note that the validity of this structural basis is not studied in the current section. It is used simply as a placeholder at this stage for the purpose of evaluating the accuracy of the kriging ROM with a fixed structural basis. A subsequent section will provide detailed examination of selection of a robust structural basis.

Using the set of sample points along with the output data, kriging surrogates are created for the stiffness matrix and thermal loads using a second-order polynomial regression model. Once the kriging models are generated, the errors associated with each are quantified. If the accuracy of the models is acceptable, they can then be used within the aerothermoelastic simulation framework. If greater accuracy is desired, more sample points are added and the kriging models are updated. The procedure for error quantification is based on that used by Crowell et al. [14]. The process involves first generating an independent set of evaluation points using LHS. Again, these evaluation points are subjected to the criteria that they must generate nodal temperatures that lie within the range 293-1500 K. The number of kriging evaluation points $n_{e}$ is chosen to be 500 in this study. For each evaluation point, the stiffness matrix and thermal load vector are calculated using both the full-order model and the kriging ROM. To quantify the error between the full-order and kriging solutions, two different error metrics are used. The first error metric is the normalized root mean-squared error. Expressed as a percentage, the NRMSE is given by

$$
\mathrm{NRMSE}=\frac{\sqrt{(1 / q) \sum_{i=1}^{q}\left(\mathrm{ROM}_{i}-\mathrm{Full}_{i}\right)^{2}}}{\operatorname{Max}(\text { Full })-\operatorname{Min}(\text { Full })} \times 100 \%
$$

where $i$ is the $i$ th output quantity, ROM represents a solution vector of the reduced-order model, Full represents a solution vector of the fullorder model, and $q$ is the total number of output quantities. Also, note that "Max" and "Min" correspond to the maximum and minimum entries, respectively, of the vector of interest. The output quantities are the entries of the generalized stiffness matrix and the physical or generalized thermal load vector, and thus $i$ is summed over all of the entries of the vector of interest in the numerator of Eq. (40). Note that the generalized stiffness matrix is reshaped into a column vector for the purpose of error analysis. The second error metric used is the $L_{\infty}$ error. Expressed as a percentage, the $L_{\infty}$ error is given by

$$
L_{\infty}=\frac{\operatorname{Max}(\mid \mathrm{ROM}-\text { Full } \mid)}{\operatorname{Max}(\text { Full })-\operatorname{Min}(\text { Full })} \times 100 \%
$$

Note that the error metrics are calculated for each evaluation case. To determine a scalar measure of error for the ROMs, both the average and maximum values of the NRMSE and $L_{\infty}$ error over all evaluation cases are examined.

To examine the error of the kriging ROMs as a function of number of sample points, kriging models for both the generalized stiffness matrix and generalized thermal load vector are created using varying numbers of sample points. Two different criteria are used in generating the set of Latin hypercube sample points. The first, denoted "maximin," aims to maximize the minimum distance between sample points over a specified number of iterations. The second, denoted "correlation," aims to minimize the correlation between sample points over a specified number of iterations. In both cases, the maximum allowable number of iterations is set to 500. The error metrics are computed for each kriging ROM over 500 evaluation cases, which are always generated using the maximin criterion over 500 iterations. Plots of the average NRMSE and $L_{\infty}$ error for the generalized stiffness ROM as a function of number of sample points are given in Figs. 14a and 14b, respectively, using both the maximin and correlation criteria. Similarly, plots of the average error for the generalized thermal load vector ROM are given in Figs. $15 \mathrm{a}$ and $15 \mathrm{~b}$, respectively, using both the maximin and correlation criteria. Due to memory requirements associated with the maximin criterion, the maximum number of sample points used with this criterion is 3000 , whereas up to 6000 are taken for the correlation 


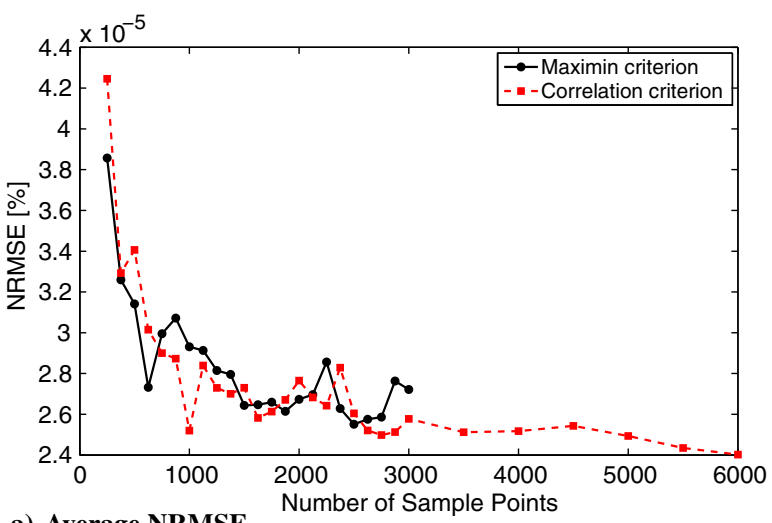

a) Average NRMSE

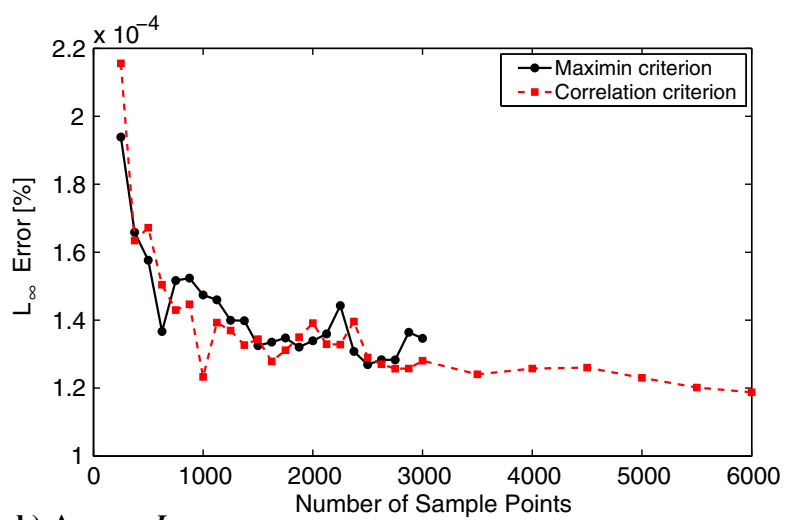

b) Average $L_{\infty}$ error

Fig. 14 Average errors for generalized stiffness matrix ROM over 500 evaluation cases.

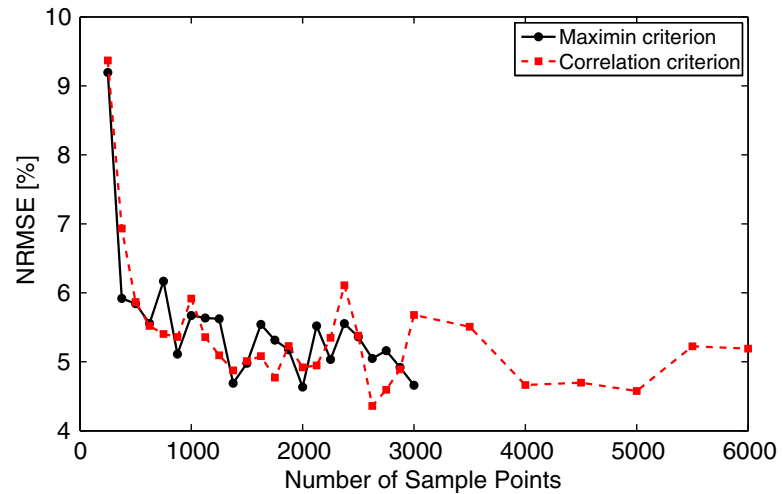

a) Average NRMSE

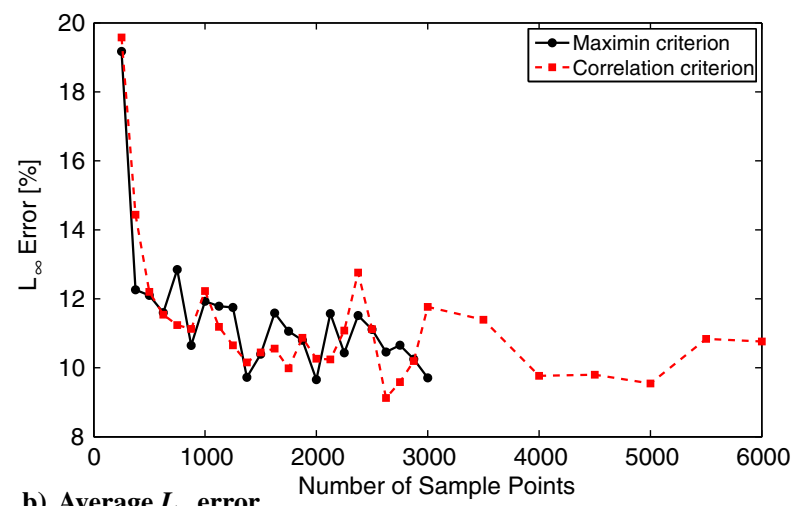

b) Average $L_{\infty}$ error

Fig. 15 Average errors for generalized thermal load vector ROM over 500 evaluation cases.

cases. As seen in the figures, there is not a significant difference between the results obtained using the maximin criterion and the correlation criterion. Due to the higher computational cost and memory requirements associated with the maximin criterion, the correlation criterion with a maximum of 500 iterations is used from this point forward. Comparing Fig. 14 with Fig. 15, it is evident that the generalized stiffness matrix ROM is significantly more accurate than the generalized thermal load vector ROM for the same number of snapshots.

To understand why the error of the generalized stiffness ROM is significantly lower than that of the generalized thermal load vector ROM, the functional dependence of the generalized stiffness matrix on the POD modal coordinates is examined. Recall that the physical stiffness matrix is the sum of the conventional stiffness matrix and geometric stiffness matrix, i.e.,

$$
K_{S}^{*}(T) \equiv K_{S}(T)+K_{G}(T)
$$

The entries of the generalized stiffness matrix are simply linear combinations of the entries of the physical stiffness matrix and do not affect the order of its dependence on temperature. The dependence of $K_{S}(T)$ and $K_{G}(T)$ on the POD modal coordinates is through the variation of material properties with temperature. In addition, $K_{G}(T)$ is also a function of $\Delta T$. The entries of the conventional stiffness matrix $K_{S}(T)$ are only dependent on the Young's modulus of the corresponding material, i.e.,

$$
K_{S, i} \propto E\left(T_{i}\right)
$$

where $i$ denotes a particular degree of freedom. The entries of the geometric stiffness matrix $K_{G}(T)$ are dependent on the Young's modulus, the coefficient of thermal expansion, and the temperature change, i.e.,

$$
K_{G, i} \propto E\left(T_{i}\right), \quad \alpha_{T}\left(T_{i}\right), \quad \Delta T_{i}
$$

where the temperature is always expressed as a linear combination of the POD basis vectors such that

$$
T_{i}=\sum_{j=1}^{r} c_{j} \varphi_{i}^{(j)}
$$

where $r$ is again the number of degrees of freedom in the reduced-order thermal model. To understand the order of the functional dependence the entries of $K_{S}(T)$ and $K_{G}(T)$ on the POD modal coordinates $c_{j}$, it is first necessary to know the order of the functional dependence of $E$ and $\alpha_{T}$ on temperature for each material in the model. This information is given in Table 3 for each of the three materials used in the model, where each entry in the table gives the order of the polynomial approximating the temperature dependence of the material property. Recall that the stiffness and thermal expansion of the insulation layer are neglected in the structural model.

Based on the information given in Table 3 , the entries of $K_{S}(T)$ can depend on, at most, the second power of $c_{j}$, and the entries of $K_{G}(T)$ can depend on, at most, the sixth power of $c_{j}$. Because the regression model used for the kriging function is quadratic, one would expect the generalized stiffness matrix to be well approximated by the kriging model if the generalized stiffness was, at most, a quadratic function of

Table 3 Orders of dependence of $E$ and $\alpha_{T}$ on temperature $^{\mathrm{a}}$

\begin{tabular}{lcc}
\hline \hline & $E(T)$ & $\alpha_{T}(T)$ \\
\hline Heat shield (René 41) & Quadratic & Cubic \\
Insulation (Min-K) & N/A & N/A \\
Skin/stiffeners (TIMETAL834) & Linear & Constant \\
\hline \hline
\end{tabular}

${ }^{a}$ N/A denotes "not applicable." 
the POD modal coordinates. It is found that, for the structure under consideration, $K_{S}(T)$ is dominant over $K_{G}(T)$, and thus $K_{S}^{*}(T)$ is essentially quadratic in $T$ with small perturbations due to geometric stiffening.

Though the average NRMSE of the generalized thermal load vector remains below 10\%, as shown in Fig. 15a, higher accuracy for the thermal loads is still desired. One potential means for improving the representation of the thermal loads is to perform the kriging interpolation on the physical thermal load vector $F_{S}^{H}$ as opposed to the generalized thermal load vector $f_{S}^{H}$. Because the number of entries in the physical thermal load vector is at a feasible level (8074), memory and computational cost issues will not come into play as they would in the case of the physical stiffness matrix. To investigate the ability of kriging to capture the entries of the physical thermal load vector, the same error analysis as used for the generalized thermal load vector is repeated on the physical one. The correlation criterion with a maximum of 500 iterations is again used to generate the sample points for the training data. Plots of the average NRMSE and $L_{\infty}$ error over the evaluation cases are given in Figs. $16 \mathrm{a}$ and $16 \mathrm{~b}$, respectively.

Comparing Fig. 16 to Fig. 15, it is observed that greater accuracy can be obtained by performing the kriging interpolation on the physical thermal load vector instead of the generalized thermal load vector. To understand why this is the case, first consider the dependence of the entries of the physical load vector on temperature. The physical thermal load vector is proportional to the Young's modulus, the coefficient of thermal expansion, and the change in temperature, i.e.,

$$
F_{S, i}^{H} \propto E\left(T_{i}\right), \alpha_{T}\left(T_{i}\right), \Delta T_{i}, \text { where } T_{i}=\sum_{j=1}^{r} c_{j} \varphi_{i}^{(j)}
$$

Based on the information presented in Table $\underline{3}$, this means that the physical thermal load vector depends on the second power of $\boldsymbol{c}$ for skin and stiffener elements and the sixth power of $\boldsymbol{c}$ for heat shield elements. To compute the generalized thermal load vector, inner products between the structural modes and the physical thermal load vector are required. The $i$ th entry of the generalized thermal load vector is given by

$$
f_{S, i}^{H}=\sum_{l=1}^{s} \phi_{l}^{(i)} F_{S, l}^{H}
$$

where $\phi_{l}^{(i)}$ denotes the $l$ th entry of the $i$ th structural reference mode, and $s$ is the total number of degrees of freedom in the structural model (8074 in this case). Whereas the physical thermal load vector depends on the second power of $c$ for skin and stiffener elements and the sixth power of $c$ for heat shield elements, the generalized thermal load vector depends on the sixth power of $\boldsymbol{c}$ for all elements because it is summed over all entries of $F_{S}^{H}$. The only caveat associated with using kriging to generate the physical load vector is that the physical thermal load vector must be premultiplied by the structural reference modes each time it is updated in order to transform to modal space. However, the computation time involved in transforming the loads from physical space to modal space is expected to be acceptable. Thus, the physical thermal load vector and not the generalized thermal load vector will be approximated with kriging in this work.

Though the accuracy of the generalized stiffness matrix and physical thermal load vector ROMs appear sufficient based on the NRMSE and $L_{\infty}$ plots given in Figs. 14 and $\underline{16}$, recall that these results are obtained by averaging the error metrics over 500 evaluation cases. As one of the objectives of this study is to bound the error of the kriging ROMs, it is necessary to investigate the worstcase error over the parameter space. As such, the maximum $L_{\infty}$ error over the 500 evaluation cases is plotted for both the generalized stiffness matrix ROM and the physical thermal load vector ROM as a function of number of sample points. This metric gives the worst-case error over all degrees of freedom over all evaluation cases. Results are given in Figs. 17a and 17b. Comparing the two figures, the worst-case error of the physical thermal load vector ROM is several orders of
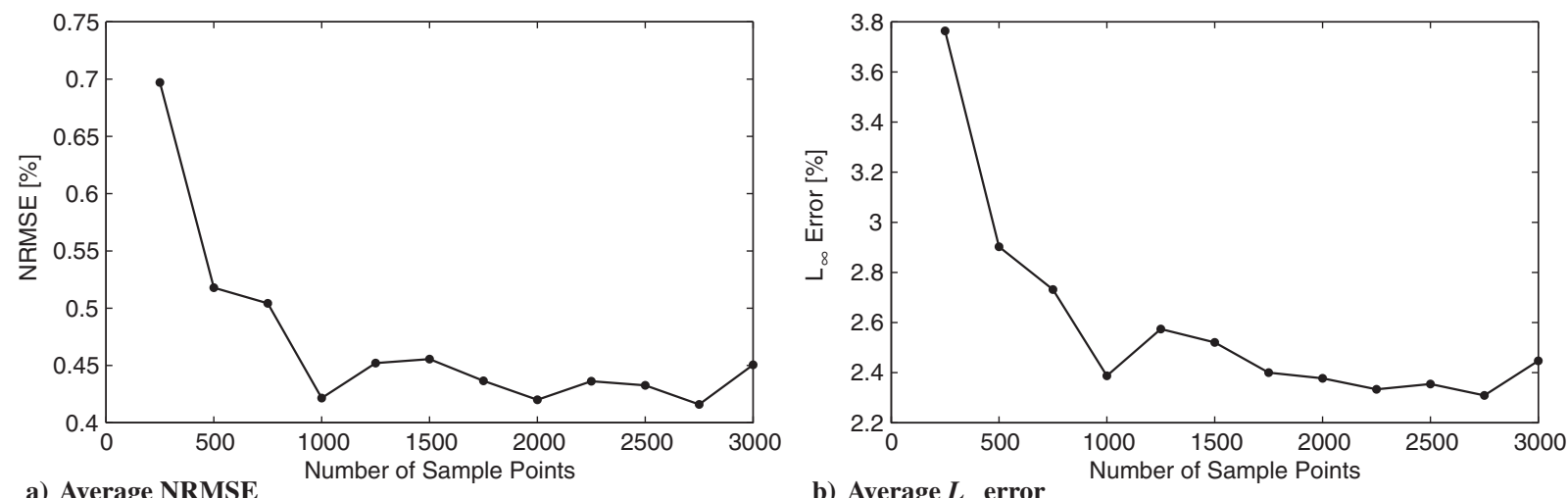

Fig. 16 Average errors for physical thermal load vector ROM over 500 evaluation cases.

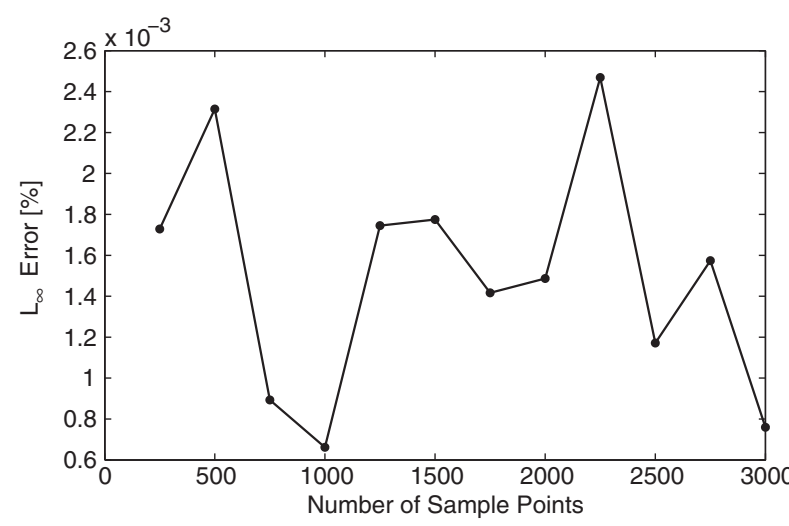

a) Generalized stiffness matrix ROM

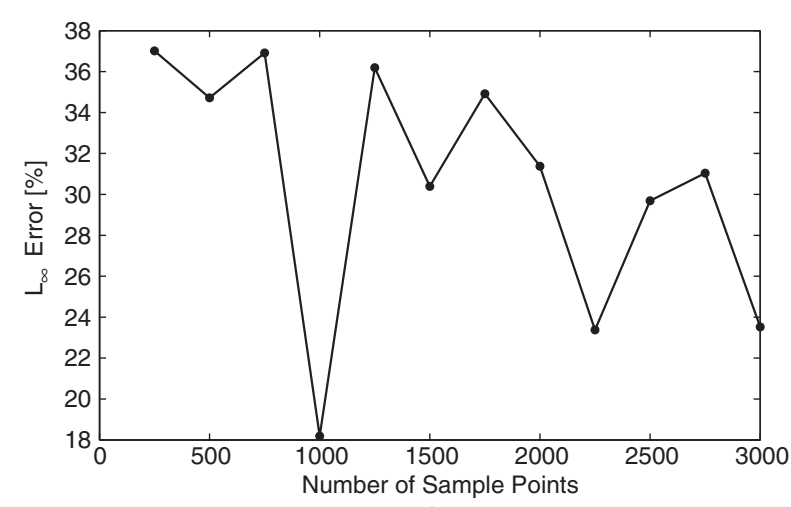

b) Physical thermal load vector ROM

Fig. 17 Maximum $L_{\infty}$ errors over 500 evaluation cases for kriging ROMs of $k_{S}^{*}(T)$ and $F_{S}^{H}(T)$ using 2nd order regression model. 


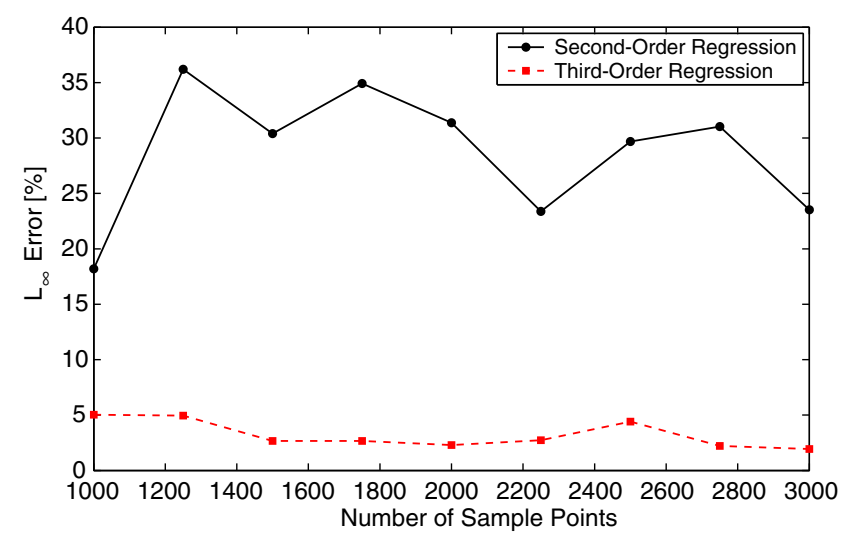

Fig. 18 Maximum $L_{\infty}$ error over 500 evaluation cases for kriging ROMs of $F_{S}^{H}(T)$ using both second- and third-order regression models.

magnitude higher than that of the generalized stiffness matrix ROM. Further investigation reveals that the maximum percentage error generally occurs at those degrees of freedom corresponding to the heat shield. This is expected, as the thermal loads for the heat shield are a higher-order function of temperature than the thermal loads for the skin and stiffeners.

Due to the higher-order dependence of the heat shield thermal loads on temperature, it is expected that the use of a higher-order regression model will improve the accuracy of the representation of the thermal loads. To examine this hypothesis, a third-order regression model is implemented for comparison against the results obtained using the second-order regression model. The maximum $L_{\infty}$ error of the physical thermal load vector ROM over 500 evaluation cases is plotted as a function of number of sample points for both the second-order and third-order regression models, as shown in Fig. 18. The figure shows significant improvement in capturing the thermal loads by moving from a second-order regression function to a third-order regression function. It is observed that, for the third-order regression function, all data points remain at or below a $5 \%$ maximum $L_{\infty}$ error over the evaluation cases.

Note that the minimum number of sample points used in Fig. 18 is 1000. This is due to the fact that there is a minimum bound on the number of sample points used to create the kriging ROM based on the number of basis functions $n_{b}$ required by the regression model. To ensure that the problem is not underconstrained, the number of sample points used in creating the kriging ROM must be equal to or greater than the number of basis functions in the regression model, $i$. e., $n_{k} \geq n_{b}$. Therefore, the order of the regression model must not be chosen to be arbitrarily high because there is a tradeoff between the order of regression and the minimum number of required sample points.

\section{Final Parameters for Kriging Models of Stiffness and Thermal Loads}

A summary of the final parameters selected for the two kriging models as well as the errors over 500 evaluation cases are given in Table 4 , where $\mathcal{O}$ denotes the order of the kriging regression model. The last three columns in the table give the CPU time required to generate the kriging training data $t_{T}$, create the kriging ROMs $t_{K}$, and evaluate the kriging ROMs $t_{E}$. Though the two ROMs use a different number of sample points to generate the models, both use 500

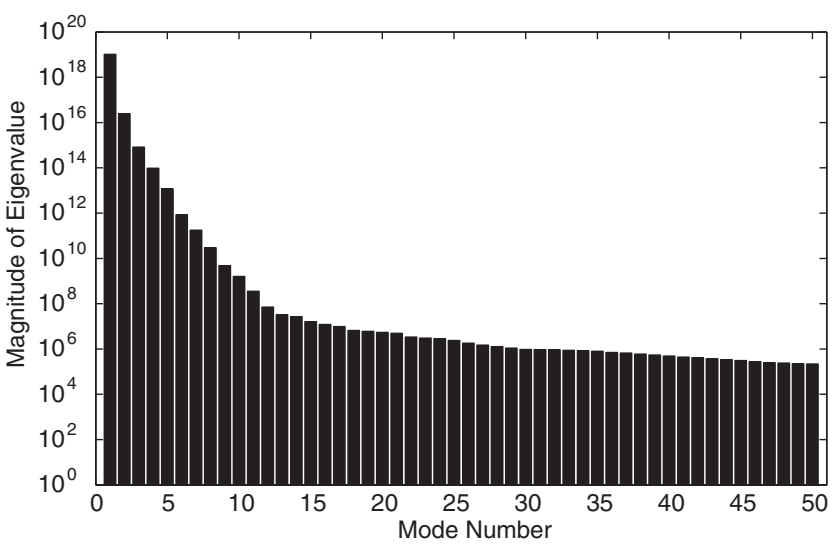

Fig. 19 Eigenvalues associated with first 50 POD modes of $F_{S}$ based on 3000 snapshots.

evaluation points for determining their accuracy. Note that $t_{E}$ is greater for the kriging model of $k_{S}^{*}(T)$ than for that of $F_{S}^{H}(T)$ due to the longer computation required to evaluate $k_{S}^{*}(T)$ at each evaluation point. For the kriging model of the generalized stiffness, 20 input variables, 500 sample points, and a second-order regression model are used. For the resulting kriging model based on the 10 free vibration modes employed, the average NRMSE and maximum $L_{\infty}$ error over 500 evaluation points are found to be 0.05 and $1.3 \%$, respectively. Because the generalized stiffness matrix is dependent on the structural basis, this kriging ROM must be updated each time the basis is changed. However, because less sample points and a lowerorder regression model are required, the computational cost of generating the kriging ROM of the generalized stiffness matrix is less than that required to generate the kriging ROM of the thermal load vector. For the kriging model of the thermal loads, 20 input variables, 5000 sample points, and a third-order regression model are used. For the resulting kriging model, the average NRMSE and maximum $L_{\infty}$ error over 500 evaluation points are found to be 0.1 and $4.7 \%$, respectively. Because kriging is used to represent the physical thermal load vector as opposed to the generalized one, this model is independent of the structural basis and does not need to be updated if the structural basis changes. As both kriging models use 20 input variables, the first 19 input variables are the POD modal coordinates of the corresponding POD modes, and the 20th input variable represents the uniform initial condition as described previously.

As the motivation for the use of the kriging ROMs is to improve the computational cost of the structural dynamic solution, the computational savings achieved via the use of these ROMs must be quantified. To do so, the full-order and reduced-order structural solutions are each run for 10 time steps. The full-order solution consists of calling Nastran to generate the equations of motion in physical space and marching the solution forward one time step. The reduced-order solution consists of using the kriging ROMs to generate the equations of motion in modal space and marching the solution forward one time step. For both the full-order and reducedorder solutions, the computation time is recorded for each of the 10 time steps. The maximum and minimum computation times are removed for each case, and the remaining eight values are averaged to determine the average computation time for the reduced-order and full-order models. The average computation time for the full-order structural model is found to be $7.22 \mathrm{~s}$, whereas that for the reduced-

Table 4 Parameters for kriging ROMs of $k_{S}^{*}(T)$ and $F_{S}^{H}(T)$

\begin{tabular}{|c|c|c|c|c|c|c|c|c|}
\hline Model & $n_{\mathrm{iv}}$ & $n_{k}$ & $\mathcal{O}$ & Average NRMSE, \% & Maximum $L_{\infty}, \%$ & $t_{T^{\mathrm{a}}}^{\mathrm{a}}, \mathrm{h}$ & $t_{K^{\mathrm{a}}}, \mathrm{h}$ & $t_{E^{\mathrm{a}}}, \mathrm{h}$ \\
\hline$\overline{k_{S}^{*}(T)}$ & 20 & 500 & Second order & 0.051 & 1.33 & 0.81 & 0.051 & $\overline{0.78}$ \\
\hline$F_{S}^{H}(T)$ & 20 & 5,000 & Third order & 0.12 & 4.68 & 5.61 & 71 & 0.55 \\
\hline
\end{tabular}

a One 2.53 GHz Intel Xeon E5540 processor with 3.0 GB RAM. 
Table 5 Parameters used for load-dependent Ritz vector case 1

\begin{tabular}{lc}
\hline \hline Parameter & Value \\
\hline Case & 1 \\
$M_{\infty}$ & 8 \\
$\alpha, \mathrm{deg}$ & 4 \\
$h, \mathrm{~km}$ & 35 \\
$\Delta t_{\mathrm{AE}}, \mathrm{s}$ & 0.1 \\
$\Delta t_{\mathrm{HT}}, \mathrm{s}$ & 0.1 \\
$\Delta t_{\mathrm{AT}}, \mathrm{s}$ & 1 \\
$T_{0}, \mathrm{~K}$ & 293 \\
\hline \hline
\end{tabular}

Table 6 Parameters for aerothermoelastic subcases used to assess error incurred due to kriging ROMs of $k_{S}^{*}(T)$ and $F_{S}^{H}(T)$

\begin{tabular}{lccccc}
\hline \hline & & & & \multicolumn{3}{c}{ Method for computing } \\
Subcase & Structural model & $n_{V}$ & $n_{F}$ & $n_{R}$ & $k_{S}^{*}(T)$ and $F_{S}^{H}(T)$ \\
\hline 1a & Full-order & N/A & N/A & N/A & N/A \\
1b & ROM & 6 & 0 & 0 & Nastran \\
1c & ROM & 6 & 0 & 0 & Kriging ROMs \\
\hline \hline
\end{tabular}

order structural model is found to be $1.42 \mathrm{~s}$, resulting in an improvement in computational cost by a factor of five. Note that, in both the full-order and reduced-order cases, the simulations are performed using one $2.53 \mathrm{GHz}$ Intel Xeon E5540 processor with 3.0 GB of RAM.

\section{E. Load-Dependent Ritz Vector Results}

As discussed in Sec. VII, the representative load vectors $F_{S}^{I}$ to be used in the load-dependent Ritz vector formulation are identified using the POD approach. Similar to the case of the POD procedure for the thermal ROM, snapshots of the structural load vector $F_{S}$ must be

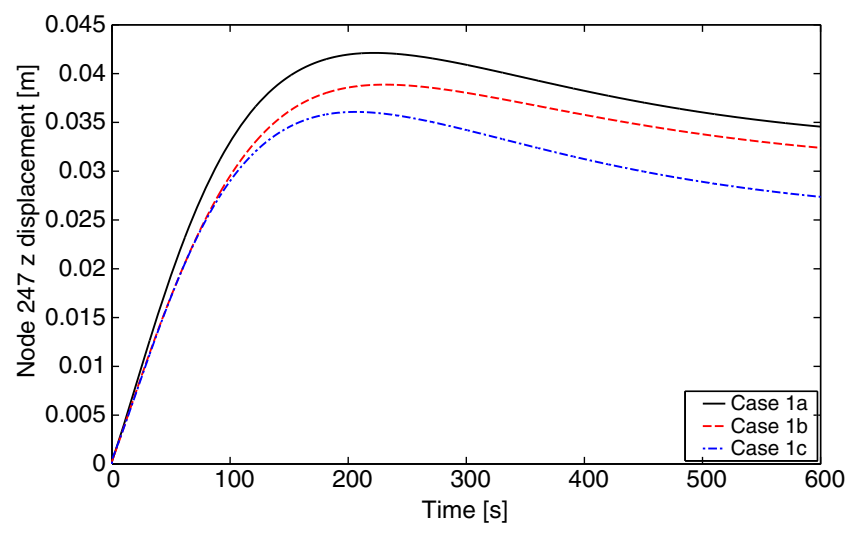

Fig. 20 Node $247 z$ displacements for subcases 1a, 1b, and 1c. collected to derive the corresponding POD basis vectors. These snapshots of the structural load include contributions due to both thermal loads and aerodynamic loads, i.e.,

$$
F_{S}(t)=F_{S}^{H}(t)+F_{S}^{A}(t)
$$

The snapshots are taken by running an aerothermoelastic simulation for a period of $3000 \mathrm{~s}$. This simulation uses the thermal ROM with the 32 previously identified thermal POD modes. For the structural dynamic solution, the full-order model is used. The flight conditions for this simulation are $M_{\infty}=6.5, \alpha=2 \mathrm{deg}$, and $h=35 \mathrm{~km}$, corresponding to the midpoints of the ranges given in Table 2. The initial temperature distribution is taken to be a uniform $293 \mathrm{~K}$. The time steps for the various solution components are as follows: $\Delta t_{\mathrm{AE}}=\Delta t_{\mathrm{HT}}=0.1 \mathrm{~s}$ and $\Delta t_{\mathrm{AT}}=1 \mathrm{~s}$. The structural load vector is stored at the end of each aerothermal time step, resulting in a total of 3000 snapshots. The eigenvalues corresponding to the first 50 POD modes are given Fig. 19.

To exercise the load-dependent Ritz vector formulation, aerothermoelastic simulations of the lifting surface model are carried out using both the full-order and reduced-order structural dynamic models. The full-order model is treated as the truth model, and its solution is obtained by solving the equations of motion in physical space given by Eq. (6). For the full-order model, the equations of motion are generated directly using Nastran. Recall that the full-order and reduced-order models are both solved using the same Newmark- $\beta$ integration scheme described in Sec. V.B to eliminate discrepancies due to different numerical integration methods. The reduced-order model consists of solving the modal equations of motion given in Eq. (9) where the structural modal matrix $\Phi_{S}$ can contain both reference free vibration modes and loaddependent Ritz vectors obtained using the formulation outlined previously. The reduced-order model can update the equations of motion at each aeroelastic time step by calling Nastran directly or by using the kriging ROMs of the generalized stiffness matrix and physical thermal load vector obtained using the parameters $n_{\mathrm{iv}}, n_{k}$, and $\mathcal{O}$ in Table 4 . Both the full-order and reduced-order models make use of the thermal ROM with the 32-mode basis described in Sec. VIII.B. For the structural ROM, the basis consists of a predetermined set of free vibration modes appended with a set of load-dependent Ritz vectors. For all studies in the current section, the number of free vibration modes $n_{V}$ is taken to be six and the mode shapes are chosen to be the first six of the 10 reference modes employed in Sec. VIII.C based on a cutoff frequency of $100 \mathrm{~Hz}$. This six-mode subset has a maximum frequency of $94.9 \mathrm{~Hz}$. The goal of this section is to assess the effect of the number of specified load vectors $n_{F}$ and the number of load-dependent Ritz vectors per specified load vector $n_{R}$ on the accuracy of the reduced-order structural dynamic model. Note that the total number of structural basis vectors is equal to $n_{V}+\left(n_{F}\right)\left(n_{R}\right)$.

To quantify the error between the full-order and reduced-order structural dynamic models, the NRMSE and $L_{\infty}$ error metrics, given in Eqs. (40) and (41), are employed. In this case, the quantities Full and $\mathrm{ROM}$ are taken to be vectors of $z$-direction displacements for
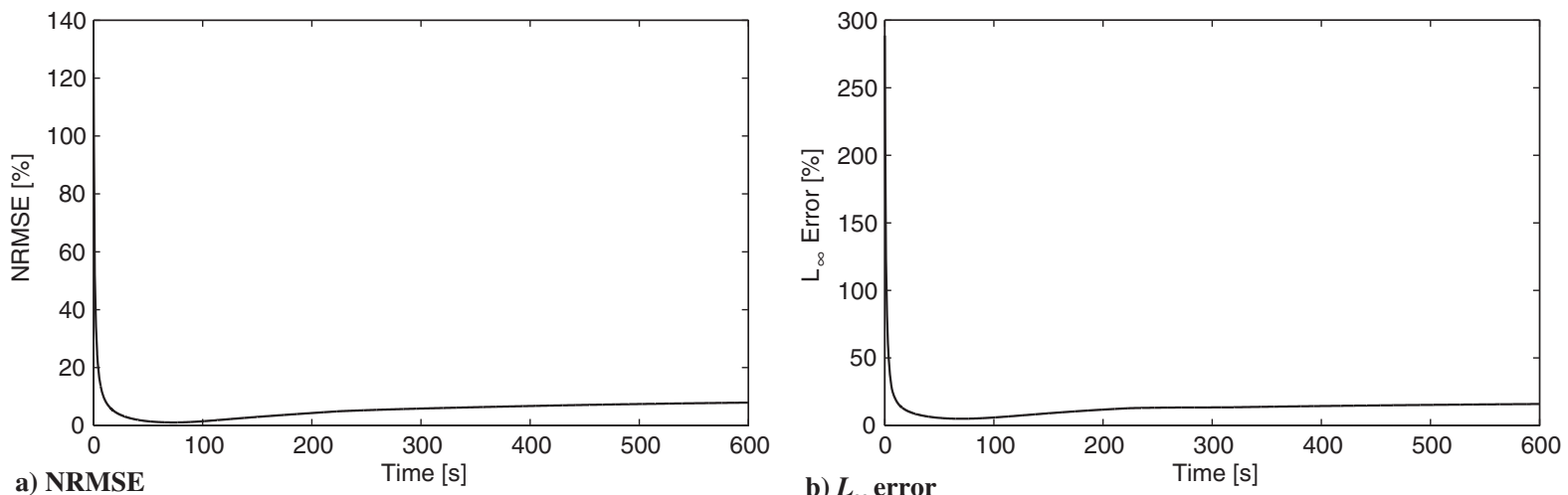

Fig. 21 Error of subcase 1c with respect to subcase 1b illustrating error incurred due to kriging ROMs of $k_{S}^{*}(T)$ and $F_{S}^{H}(T)$. 
Table 7 Parameters for aerothermoelastic subcases used to assess effect of load-dependent Ritz vectors on structural ROM

nodes at the outer mold line from the full-order and reduced-order models, respectively. The parameters for the aerothermoelastic simulations used in this part of the study are given in Table $\underline{5}$. The total simulation time for this case is chosen to be $600 \mathrm{~s}$.

Before assessing the effect of load-dependent Ritz vectors, the impact of using kriging ROMs to generate $k_{S}^{*}(T)$ and $F_{S}^{H}(T)$ on the accuracy of the solution must first be addressed. Doing so provides insight into how much of the solution error can be attributed to error associated with the kriging ROMs as opposed to error associated with the reduced structural basis. To accomplish this, aerothermoelastic simulations are carried out for the flight conditions of case 1 for three different subcases that are summarized in Table 6 . For subcase 1a, the full-order structural model is used in the simulation and the results are treated as the truth model. Subcase $1 \mathrm{~b}$ consists of using the structural ROM with only the six free vibration modes (i.e., $n_{V}=6, n_{F}=0$, $n_{R}=0$ ), and using Nastran to compute $k_{S}^{*}(T)$ and $F_{S}^{H}(T)$ at each aeroelastic time step. Subcase 1c consists of using the structural ROM again with the six free vibration modes, but employing the kriging ROMs to compute $k_{S}^{*}(T)$ and $F_{S}^{H}(T)$. It is expected that the six-mode basis will not adequately capture the structural response represented by the full-order model. However, the goal of subcases $1 \mathrm{a}-1 \mathrm{c}$ is not to assess the error associated with the reduced structural basis but rather to evaluate the error incurred by employing the kriging models to approximate $k_{S}^{*}(T)$ and $F_{S}^{H}(T)$.

To assess the response levels, the $z$-direction displacements of node 247 (located at the mid-chord of the tip on the bottom surface) are plotted for each of the three subcases given in Table $\underline{6}$. Results are given in Fig. 20. To quantify the error incurred due solely to the kriging ROMs of $k_{S}^{*}(T)$ and $F_{S}^{H}(T)$, the NRMSE and $L_{\infty}$ error metrics are computed for subcase $1 \mathrm{c}$ with respect to subcase $1 \mathrm{~b}$ and are given in Figs. 21a and 21b, respectively. The error metrics show an initial high error, which is due to the fact that the simulation begins with the structure in the undeformed configuration. Therefore, the structural displacements are small in the initial part of the transient, which results in a small denominator in Eqs. (40) and (41), and therefore a large error early in the transient. However, within $\overline{10} \mathrm{~s}$ into the transient, the NRMSE and $L_{\infty}$ error decrease to below 10 and
$20 \%$, respectively, and remain below these values for the remainder of the simulation.

With the error due to the kriging ROMs of $k_{S}^{*}(T)$ and $F_{S}^{H}(T)$ quantified, the next step is to assess the effect of inclusion of loaddependent Ritz vectors on the accuracy of the structural ROM. For all cases from this point forward, the kriging ROMs of $k_{S}^{*}(T)$ and $F_{S}^{H}(T)$ are used within the structural ROM. The goal of this aspect of the study is to assess the solution accuracy as a function of $n_{F}$ and $n_{R}$. A summary of the subcases used to perform this assessment is given in Table 7 . Note that the errors for these subcases are computed with respect to subcase 1a in Table $\underline{6}$; therefore, they include both error due to the kriging ROMs as well as error due to structural basis truncation.

Plots of the NRMSE and $L_{\infty}$ errors of subcases $1 \mathrm{c}-1 \mathrm{f}$ with respect to subcase $1 \mathrm{a}$ are given in Figs. $22 \mathrm{a}$ and $22 \mathrm{~b}$, respectively. Examining these figures, it is observed that the inclusion of loaddependent Ritz vectors in addition to the six free vibration modes results in a noticeable improvement in the structural ROM. It should be noted that there is inherent error in the structural ROM due to the use of kriging to approximate $k_{S}^{*}(T)$ and $F_{S}^{H}(T)$, and thus the overall error of the structural ROM cannot be reduced to zero. Comparing Fig. 22 to Fig. 21, it can be observed that the error due to basis truncation has been reduced and the errors shown in Fig. 22 approach or surpass those shown in Fig. 21, especially for subcases $1 \mathrm{e}$ and 1f. Comparing subcase $1 \mathrm{~d}$ to subcases $1 \mathrm{e}$ and $1 \mathrm{f}$, it is observed that the inclusion of only one load-dependent Ritz vector in the basis does not provide the level of accuracy obtained by including multiple load-dependent Ritz vectors. The greatest difference in $L_{\infty}$ error beyond $10 \mathrm{~s}$ between subcases $1 \mathrm{e}$ and $1 \mathrm{f}$ occurs at $78.1 \mathrm{~s}$, with the $L_{\infty}$ error of subcase $1 \mathrm{e}$ being $11.2 \%$ lower than that of subcase 1f. This is not surprising, as the structural response is dominated by the slowly changing thermal loads for this case; therefore, inertial effects are not significant. The maximum improvement in $L_{\infty}$ error of subcase 1e with respect to subcase 1c beyond $10 \mathrm{~s}$ is $45 \%$ and occurs at $590.1 \mathrm{~s}$. Subcase 1e gives an average improvement in $L_{\infty}$ error of $38 \%$ over subcase 1c, demonstrating the advantage of using load-dependent Ritz vectors.
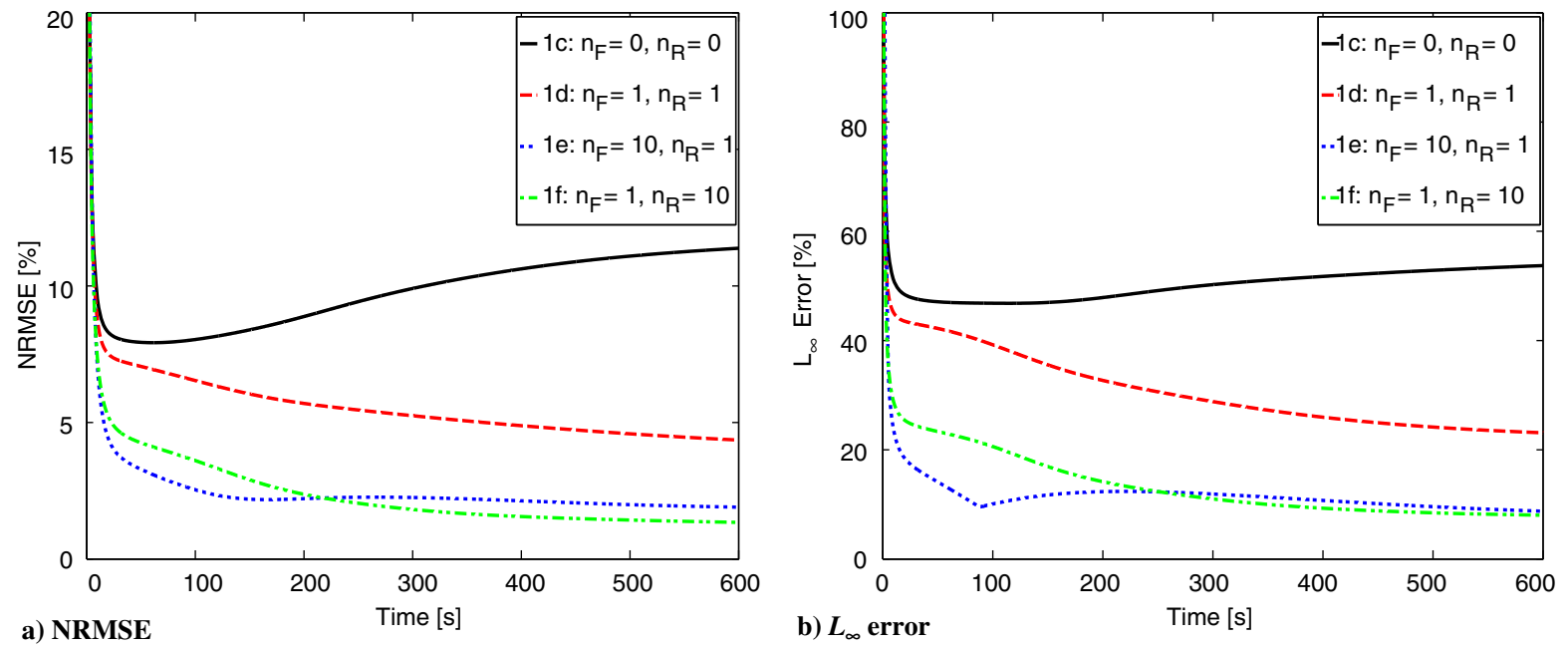

Fig. 22 Total error of structural ROMs (subcases 1c-1f) with respect to full-order structural model (subcase 1a) using different structural bases. 


\section{Conclusions}

A comprehensive study of structural dynamic response simulation within an aerothermoelastic analysis framework has been conducted with the goals of improving the computational efficiency and accuracy of the structural dynamic reduced-order model (ROM). The study addressed two main modeling areas associated with structural dynamic reduced-order modeling in an aerothermoelastic framework:

1) A surrogate modeling technique was implemented to allow for direct updates to the temperature-dependent generalized stiffness matrix and thermal loads.

2) Load-dependent Ritz vectors were used to improve the accuracy of the structural dynamic ROM.

The first part of this study focused on the development of a krigingbased method for directly updating the generalized stiffness matrix and thermal loads based on a given temperature distribution. The temperature distribution was parameterized in terms of the thermal proper orthogonal decomposition (POD) modal coordinates, as this allowed for a small number of input variables to be used to represent the high-dimensional temperature vector. A methodology was described for bounding the POD modal coordinates based on a set of parallel aerothermoelastic simulations. A series of studies was used to guide the selection of various parameters involved in the generation of the kriging surrogates. The resulting ROMs of the generalized stiffness matrix and physical thermal load vector were found to have maximum $L_{\infty}$ errors of 1 and $5 \%$, respectively, over 500 evaluation points, indicating good agreement with the full-order model. Comparison of computation times showed that the structural ROM with the kriging models improved the computational cost of the structural dynamic response solution by a factor of five with respect to the full-order model.

To improve the accuracy of the structural ROM, basis augmentation using load-dependent Ritz vectors was examined. Load-dependent Ritz vectors are advantageous in that they allow for incorporation of the expected spatial dependence of the structural loads into the modal matrix. To determine the representative load vectors to be used in generating the load-dependent Ritz vectors, proper orthogonal decomposition was employed based on snapshots of the structural load vector from representative simulations. Application of the methodology to a hypersonic cruise trajectory demonstrated an average improvement in $L_{\infty}$ error of $38 \%$ for one case compared with a case using only free vibration modes. These results indicated that basis augmentation can be advantageous in cases where the structural response contains a quasi-static component as a result of thermal loads.

\section{Acknowledgments}

This work was supported by the Michigan-U.S. Air Force Research Laboratory Collaborative Center in Control Science under grant number FA 8650-07-2-3744 (U.S. Air Force Research Laboratory/Air Vehicles Directorate) with Michael Bolender as Program Manager. Opinions, interpretations, conclusions, and recommendations are those of the authors and are not necessarily endorsed by the U.S. Government.

\section{References}

[1] Anderson, J. D., Jr., Hypersonic and High-Temperature Gas Dynamics, McGraw-Hill, New York, 1989, pp. 214-215.

-[2] Perez, R., Wang, X. Q., and Mignolet, M. P., "Nonlinear Reduced-Order Models for Thermoelastodynamic Response of Isotropic and Functionally Graded Panels," AIAA Journal, Vol. 49, No. 3, 2011, pp. 630-641. doi:10.2514/1.J050684

[3] Perez, R., Wang, X. Q., and Mignolet, M. P., "Steady and Unsteady Nonlinear Thermoelastodynamic Response of Panels by Reduced Order Models," 51st AIAA/ASME/ASCE/AHS/ASC Structures, Structural Dynamics, and Materials Conference, AIAA Paper 2010-2724, 2010.

[4] Kim, K., Wang, X. Q., and Mignolet, M. P., "Nonlinear Reduced Order Modeling of Isotropic and Functionally Graded Plates," 49th AIAA/ ASME/ASCE/AHS/ASC Structures, Structural Dynamics, and Materials Conference, AIAA Paper 2008-1873, 2008.
[5] Rizzi, S. A., and Przekop, A., "System Identification-Guided Basis Selection for Reduced-Order Nonlinear Response Analysis," Journal of Sound and Vibration, Vol. 315, No. 3, 2008, pp. 467-485. doi:10.1016/j.jsv.2007.12.031

[6] Przekop, A., and Rizzi, S. A., "Efficient Modal Basis Selection Criteria for Reduced-Order Nonlinear Simulation," Proceedings of the 7th European Conference on Structural Dynamics, European Assoc. for Structural Dynamics, Southampton, England, U.K., July 2008, Paper E69.

[7] Rizzi, S. A., and Przekop, A., "POD/MAC-Based Modal Basis Selection for a Reduced Order Nonlinear Response Analysis," Proceedings of the Euromech Colloquium 483, Geometrically NonLinear Vibrations of Structures, European Mechanics Soc., July 2007, Paper 21.

[8] Guo, X., and Przekop, A., "Energy-Based Modal Basis Selection Procedure for Reduced-Order Nonlinear Simulation," 51st AIAA/ ASME/ASCE/AHS/ASC Structures, Structural Dynamics, and Materials Conference, AIAA Paper 2010-2796, 2010.

- [9] Chelidze, D., and Zhou, W., "Smooth Orthogonal DecompositionBased Vibration Mode Identification," Journal of Sound and Vibration, Vol. 292, Nos. 3-5, 2006, pp. 461-473. doi:10.1016/j.jsv.2005.08.006

[10] Falkiewicz, N. J., and Cesnik, C. E. S., "A Reduced-Order Modeling Framework for Integrated Thermo-Elastic Analysis of Hypersonic Vehicles," 50th AIAA/ASME/ASCE/AHS/ASC Structures, Structural Dynamics, and Materials Conference, AIAA Paper 2009-2308, 2009.

[11] Falkiewicz, N. J., Cesnik, C. E. S., Bolender, M. A., and Doman, D. B., "Thermoelastic Formulation of a Hypersonic Vehicle Control Surface for Control-Oriented Simulation," 2009 AIAA Guidance, Navigation, and Control Conference, AIAA Paper 2009-6284, 2009.

[12] Falkiewicz, N. J., and Cesnik, C. E. S., "Proper Orthogonal Decomposition for Reduced-Order Thermal Solution in Hypersonic Aerothermoelastic Simulations," AIAA Journal, Vol. 49, No. 5 , May 2011, pp. 994-1009. doi:10.2514/1.J050701

[13] Falkiewicz, N. J., Cesnik, C. E. S., Crowell, A. R., and McNamara, J. J., "Reduced-Order Aerothermoelastic Framework for Hypersonic Vehicle Control Simulation," AIAA Journal, Vol. 49, No. 8, Aug. 2011, pp. $1625-1646$. doi:10.2514/1.J050802

[14] Crowell, A. R., McNamara, J. J., Kecskemety, K. M., and Goerig, T. W., "A Reduced Order Aerothermodynamic Modeling Framework for Hypersonic Aerothermoelasticity," 51st AIAA/ASME/ASCE/AHS/ASC Structures, Structural Dynamics, and Material Conference, AIAA Paper 2010-2969, 2010.

[15] Sirovich, L., "Turbulence and Dynamics of Coherent Structures, Part I: Coherent Structures," Quarterly of Applied Mathematics, Vol. 45, Oct. 1987, pp. 561-571.

[16] Craig, R. R., and Kurdila, A. J., Fundamentals of Structural Dynamics, 2nd ed., Wiley, Hoboken, NJ, 2006, p. 228.

[17] MSC. Nastran Basic Dynamic Analysis User's Guide: Version 68, MSC. Software Corp., Santa Ana, CA, 2004, p. 151.

[18] Sacks, J., Welch, W. J., Mitchell, T. J., and Wynn, H. P., "Design and Analysis of Computer Experiments," Statistical Science, Vol. 4, No. 4, 1989, pp. 409-423. doi:10.1214/ss/1177012413

-[19] Simpson, T. W., Mauery, T. M., Korte, J. J., and Mistree, F., "Kriging Models for Global Approximation in Simulation-Based Multidisciplinary Design Optimization," AIAA Journal, Vol. 39, No. 12, Dec. 2001, pp. 2233-2241. doi:10.2514/2.1234

[20] Sasena, M. J., "Flexibility and Efficiency Enhancements for Constrained Global Design Optimization with Kriging Approximations," Ph.D. Dissertation, Univ. of Michigan, Ann Arbor, MI, 2002.

[21] Wilson, E. L., Yuan, M.-W., and Dickens, J. M., "Dynamic Analysis by Direct Superposition of Ritz Vectors," Earthquake Engineering and Structural Dynamics, Vol. 10, No. 6, Nov.-Dec. 1982, pp. 813-821. doi:10.1002/(ISSN)1096-9845

[22] Pendleton, E., Moster, G., and Keller, D., "Transonic Aeroelastic Models of Highly Swept Hypersonic Lifting Surfaces," Journal of Aircraft, Vol. 32, No. 6, Nov.-Dec. 1995, pp. 1169-1176. doi: $10.2514 / 3.46860$

[23] Oppenheimer, M. W., and Doman, D. B., "A Hypersonic Vehicle Model Developed with Piston Theory," 2006 AIAA Atmospheric Flight Mechanics Conference, AIAA Paper 2006-6637, 2006.

[24] McNamara, J. J., Friedmann, P. P., Powell, K. G., Thuruthimattam, B. J., and Bartels, R. E., "Aeroelastic and Aerothermoelastic Behavior in Hypersonic Flow," AIAA Journal, Vol. 46, No. 10, Oct. 2008, 
pp. 2591-2610.

doi: $10.2514 / 1.36711$

[25] Ellis, D., Pagel, L., and Schaeffer, D., "Design and Fabrication of a Radiative Actively Cooled Honeycomb Sandwich Structural Panel for a Hypersonic Aircraft," NASA TR NASA-CR-2957, March 1978.
[26] Metallic Materials and Elements for Aerospace Vehicle Structures, U.S. Dept. of Defense MIL-HDBK-5H, Dec. 1998, pp. 6-83, 6-84, 6-85, 6-88.

[27] Welsch, G., Boyer, R., and Collings, E. W., Materials Properties Handbook: Titanium Alloys, ASM International, Materials Park, OH, 1994, pp. 439-440. 
This article has been cited by:

1. Ryan Klock, Carlos E. Cesnik. Aeroelastic Stability of High-Speed Cylindrical Vehicles . [Citation] [PDF] [PDF Plus] 